\title{
Schrödinger operators in the twentieth century*
}

\author{
Barry Simon ${ }^{\text {a) }}$ \\ Division of Mathematics 253-37, California Institute of Technology, \\ Pasadena, California 91125-3700
}

(Received 4 February 2000; accepted for publication 24 February 2000)

This paper reviews the past fifty years of work on spectral theory and related issues in nonrelativistic quantum mechanics. (c) 2000 American Institute of Physics. [S0022-2488(00)01906-X]

\section{CONTENTS}

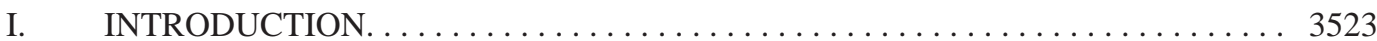

II. MATHEMATICAL TOOLS AND ISSUES. . . . . . . . . . . . . . . . . . . . . 3524

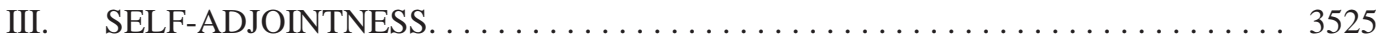

IV. PROPERTIES OF EIGENFUNCTIONS, GREEN'S FUNCTIONS, SEMIGROUPS,

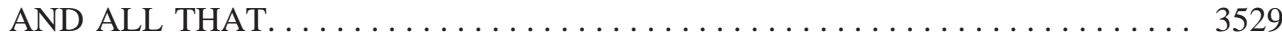

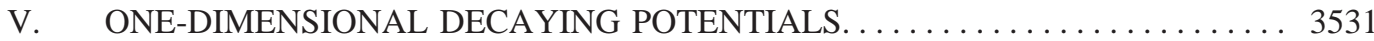

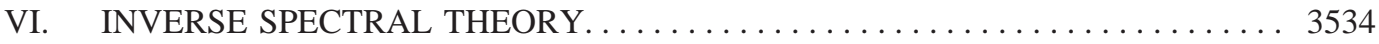

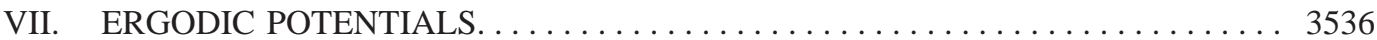

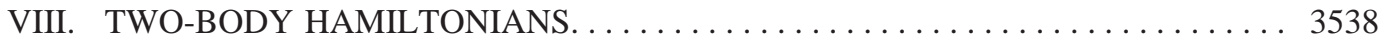

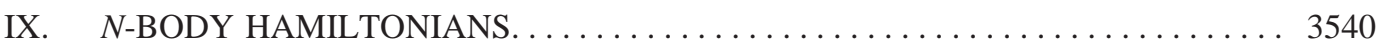

X. CONSTANT ELECTRIC AND MAGNETIC FIELDS . . . . . . . . . . . . . . 3543

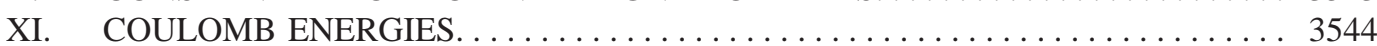

XII. EIGENVALUE PERTURBATION THEORY . . . . . . . . . . . . . . . . . . . . . 3545

XIII. RESONANCES. . . . . . . . . . . . . . . . . . . . . . . . . . . . . . . . . . . . 3546

XIV. THE QUASICLASSICAL LIMIT. . . . . . . . . . . . . . . . . . . . . . . . . 3547

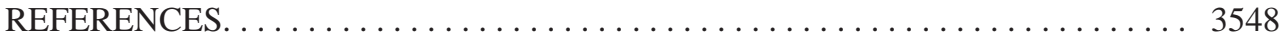

\section{INTRODUCTION}

The twentieth century is the century of science. In a century that has seen special and general relativity, quantum electrodynamics and chromodynamics, a total revamping of our understanding of molecules and of the cosmos, plate tectonics, and the rise of microbiology, one can make the case that the most spectacular scientific development was the discovery of nonrelativistic quantum mechanics in the first quarter of the century. Its aftermath not only changed the physicist's view of matter, but it set the stage for the revolutions in chemistry, our understanding of stars, biology, and practical electronics.

In what is one of the more striking cases of serendipity, just as Heisenberg and Schrödinger were discovering the "new" quantum theory, von Neumann was developing the theory of unbounded self-adjoint operators and Weyl the representations of compact Lie groups-two subjects of great relevance to the mathematics underlying nonrelativistic quantum mechanics. In short order they produced books (von Neumann ${ }^{271}$ and $\mathrm{Weyl}^{275}$ ) that used this mathematics to give a mathematical foundation to the framework of quantum mechanics. With later additions, notably by Bargmann, Wigner, and Mackey, the basic foundations are mathematically firm.

This is analogous to having formulated classical mechanics as Hamiltonian flows on symplec-

*Dedicated to Tosio Kato (1917-1999), father of the modern theory of Schrödinger operators.

${ }^{a)}$ Electronic mail: bsimon@caltech.edu 
tic manifolds. What remains is what might be called the second-level foundations-existence of solutions of the time-dependent Schrödinger equation (which is equivalent to self-adjointness of these operators) and general qualitative issues in dynamics. It is this subject, essentially born 50 years ago, that I will review here. The subject matter is vast with hundreds of contributors and thousands of papers. Each section of this paper is a proxy for what deserves a book or at least a very long review article. In attempting to overview such a vast area in a few pages, I have had to focus on the high points. No proofs are given and I have settled for usually quoting the initial or especially significant papers. I have no doubt that I have left out some important papers, and if so, I ask the forgiveness of the reader (and their authors!).

To keep this paper a reasonable size, I have focused almost entirely on the general basics of Schrödinger operators and some simple applications to atomic and molecular Hamiltonians. That means, among other areas, I have not considered general second-order operators on $\mathbb{R}^{n}$ and on general manifolds (but see Davies and Safarov, ${ }^{57}$ Davies, ${ }^{55}$ and Kenig ${ }^{154}$ ) nor have I considered some of the detailed papers on perturbations of Hamiltonians with periodic potential (see, e.g., Deift and Hempel ${ }^{58}$ and Gesztesy and Simon ${ }^{91}$ ) nor the extensive literature on Dirac operators nor the considerable work on Schrödinger operators in a bounded region with some boundary conditions including subtle results on what happens at irregular boundary points (see Davies ${ }^{55}$ ) nor the results on phenomena like the quantum Hall effect that apply and extend the general theory to results in condensed matter physics. While there are a few results about $-\Delta+V$ for cases where $V(x) \rightarrow \infty$ as $|x| \rightarrow \infty$, again there is a large literature we will not attempt to review. While Sec. $\mathrm{X}$ has a brief discussion of constant magnetic field, we have not attempted to discuss the recent extensive literature on nonconstant magnetic fields.

There is a companion piece to this one on open problems. ${ }^{260}$

\section{MATHEMATICAL TOOLS AND ISSUES}

The mathematics most relevant to the modern theory of Schrödinger operators is functional, real, harmonic, and complex analysis. In this section, we will briefly set the stage to fix notation. For more details, see Reed and Simon. ${ }^{214,211}$

Quantum Hamiltonians are unbounded operators, defined on a dense subspace rather than the whole Hilbert space. Physics books tend to emphasize the symmetry ("Hermiticity") of the Hamiltonian; that is, that $\langle H \varphi, \psi\rangle=\langle\varphi, H \psi\rangle$ for all $\varphi, \psi$ in $D(H)$. But more important is a property called self-adjointness. The adjoint $H^{*}$ of an operator $H$ is defined to be the maximal operator so that $\left\langle H^{*} \varphi, \psi\right\rangle=\langle\varphi, H \psi\rangle$ for all $\psi \in D(H), \varphi \in D\left(H^{*}\right)$. Hermiticity says only that $H^{*}$ is an extension of $H$.

We say $H$ is self-adjoint if $H=H^{*}, H$ is called essentially self-adjoint if and only if $H$ is symmetric and has a unique self-adjoint extension. This holds if and only if $H^{*}$ is self-adjoint. Self-adjointness is important in the first place because if $H$ is self-adjoint, one can form the unitary group $e^{-i t H}$ and so solve $i \dot{\varphi}_{t}=H \varphi_{t}$ (as $\varphi_{t}=e^{-i t H} \varphi$ ) for initial conditions $\varphi \in D(H)$. Indeed, Stone's theorem says that any one-parameter continuous unitary group is associated with a selfadjoint operator. Second, self-adjointness implies the spectral theorem. There is for each Borel set $A \subset \mathbb{R}$, a projection, $E_{A}(H)$, so that $H=\int \lambda d E_{\lambda}$ and $e^{-i t H}=\int e^{-i t H} d E_{\lambda}$. One defines spectral measures $d \mu_{\varphi}^{H}$ by

$$
\mu_{\varphi}^{H}(A)=\left(\varphi, E_{A}(H) \varphi\right)
$$

so that

$$
\int e^{-i t \lambda} d \mu_{\varphi}^{H}(\lambda)=\left(\varphi, e^{-i t H} \varphi\right)
$$




$$
\int \frac{d \mu_{\varphi}^{H}(\lambda)}{\lambda-z}=\left(\varphi,(H-z)^{-1} \varphi\right)
$$

$\sigma(H)$, the spectrum of $H$, is precisely $\cup_{\varphi} \operatorname{supp}\left(d \mu_{\varphi}^{H}\right)$.

Much of what we discuss in this paper involves two distinct decompositions of the spectrum of $H$. The first is

$\sigma_{\mathrm{disc}}(H)=\{\lambda \mid \lambda$ is an eigenvalue of finite multiplicity and an isolated point of $\sigma(H)\}$

$$
\sigma_{\text {ess }}(H)=\sigma(H) \backslash \sigma_{\text {disc }}(H) .
$$

Equivalently, $\lambda \in \sigma_{\text {disc }}(H)$ if and only if for some $\varepsilon>0, \operatorname{dim} E_{(\lambda-\varepsilon, \lambda+\varepsilon)}(H)$ is finite and for all $\varepsilon>0, E_{(\lambda-\varepsilon, \lambda+\varepsilon)}(H) \neq 0 . \sigma_{\text {disc }}(H)$ captures the notion of bound states.

The second breakup involves the fact that any measure $d \mu$ on $\mathrm{R}$ has a decomposition

$$
d \mu=d \mu_{\mathrm{pp}}+d \mu_{\mathrm{ac}}+d \mu_{\mathrm{sc}},
$$

where $d \mu_{\mathrm{pp}}$ is a pure point measure (sum of delta functions), $d \mu_{\mathrm{ac}}$ is $F(\lambda) d \lambda$, with $F$ a nonnegative locally integrable density, and $d \mu_{\mathrm{sc}}$ is a singular continuous measure (like the Cantor measure). I will define $\sigma_{\mathrm{pp}}(H)$ to be the set of eigenvalues of $H$; it is not the union of the supports of $\mu_{\mathrm{pp}}$ because it may not be closed

$$
\begin{aligned}
& \sigma_{\mathrm{ac}}(H)=\bigcup_{\varphi} \operatorname{supp}\left(d \mu_{\varphi}^{H}\right)_{\mathrm{ac}}, \\
& \sigma_{\mathrm{ac}}(H)=\bigcup_{\varphi} \operatorname{supp}\left(d \mu_{\varphi}^{H}\right)_{\mathrm{sc}} .
\end{aligned}
$$

One often defines a refined set $\Sigma_{\mathrm{ac}}$ with $\bar{\Sigma}_{\mathrm{ac}}=\sigma_{\mathrm{ac}}(H)$, the essential support of the ac measure. Basically, the essential support of the a.c. measure $F(\lambda) d \lambda$ is $\{\lambda \mid F(\lambda) \neq 0\}$. It is defined modulo sets of Lebesgue measure zero. $\Sigma_{\text {ac }}$ is the union of the essential support of $\left(d \mu_{\varphi}^{H}\right)_{\text {ac }}$ over a countable dense set of $\varphi$ s.

\section{SELF-ADJOINTNESS}

The theory of Schrödinger operators was born with Kato's famous self-adjointness theorem for atomic Hamiltonians. His theorem abstracted states the following:

Theorem III.1: $\left(\mathrm{Kato}^{144}\right)$ Let $\mathcal{H}=L^{2}\left(\mathbb{R}^{3 N}\right)$ where $x \in \mathbb{R}^{3 N}$ is written $\left(x_{1}, \ldots, x_{N}\right)$ with $x_{i}$ $\in \mathbb{R}^{3}$. Let $\Delta_{i}$ be the Laplacian in $x_{i}$ and let $V_{i}, V_{i j}$ be functions on $\mathbb{R}^{3}$ in $L^{2}\left(\mathbb{R}^{3}\right)+L^{\infty}\left(\mathbb{R}^{3}\right)$. Let

$$
\begin{gathered}
H_{0}=-\sum_{i=1}^{N}\left(2 \mu_{i}\right)^{-1} \Delta_{i}, \\
V=\sum_{i=1}^{N} V_{i}\left(x_{i}\right)+\sum_{i<j} V_{i j}\left(x_{i}-x_{j}\right)
\end{gathered}
$$

and let $H=H_{0}+V$. Then $H$ defined on $D\left(H_{0}\right)$ is self-adjoint and is essentially self-adjoint on $C_{0}^{\infty}\left(\mathbb{R}^{3 N}\right)$.

Remarks:

(1) See Reed and Simon ${ }^{211}$ for a proof.

(2) The basic idea of the proof is a perturbation theoretic one. There is a general theorem (the Kato-Rellich theorem) that if $A$ is a self-adjoint operator and $B$ is a symmetric operator with $D(B) \supset D(A)$ and for some $\alpha<1$ and $\beta>0$ and all $\varphi \in D(A)$, that 


$$
\|B \varphi\| \leqslant \alpha\|A \varphi\|+\beta\|\varphi\|,
$$

then $A+B$ is self-adjoint on $D(A)$ and essentially self-adjoint on any domain of essential selfadjointness for $A$. If (III.3) holds, we will say $B$ is $A$ bounded. The infimum over all $\alpha$ is called the relative bound of $B$ with respect to $A$.

(3) If one looks at a general bound of type (III.3) with $\alpha<1$ where $A=-\Delta$ on $L^{2}\left(\mathbb{R}^{k}\right)$ and $B$ is multiplication by $V$, then in terms of requirements that $V \in L_{\text {loc }}^{p}\left(\mathbb{R}^{k}\right)$, one needs

$$
\begin{gathered}
p \geqslant 2 \quad k=1,2,3 \\
p>2 \quad k=4 \\
p \geqslant \frac{k}{2} \quad k \geqslant 5
\end{gathered}
$$

by using Sobolev estimates (see, e.g., Cycon et al. ${ }^{53}$ ).

(4) If $k=3 N$ and we use only the $L^{p}$ requirements of Remark 3, Coulomb potentials stop working already at $N=2$. Thus, for Kato's theorem, it is critical to use Sobolev estimates in subsets of variables as Kato did.

An industry developed in understanding when $-\Delta+V$ is essentially self-adjoint on $C_{0}^{\infty}\left(\mathbb{R}^{n}\right)$. An illustrative example is

Example: Let $H=-\Delta-c|x|^{-2}$ on $C_{0}^{\infty}\left(\mathbb{R}^{k}\right)$ with $n \geqslant 5$ (needed for $H \varphi \in L^{2}$ for all $\varphi$ $\left.\in C_{0}^{\infty}\left(R^{k}\right)\right)$. Then it can be seen (Reed and Simon, ${ }^{211}$ Example 4 in Sec. X.2) that if $c>c_{0}=(n$ $-4) n / 4$, then $H$ is not self-adjoint on $C_{0}^{\infty}$. This is a quantum analog of the classical fact that if $V=-c|x|^{-2}$ for any $c>0$, a set of initial conditions of positive measure falls into $x=0$ in finite time $\left(c_{0}>0\right.$ is a reflection of an uncertainty principle repulsion).

This example shows that for pure $L^{p}$ requirements, one cannot do better than (III.4) since $|x|^{-2} \in L^{p}+L^{\infty}$ if $p<k / 2$. But it turns out this is only so if $V$ is allowed to have any sign. For $V \geqslant 0$, one can do much better. The best result of this genre is

Theorem III.2: (Leinfelder and Simader $\left.{ }^{173}\right)$ Let $V \geqslant 0, V \in L_{\mathrm{loc}}^{2}\left(\mathbb{R}^{k}\right),\left\{a_{j}\right\}_{j=1}^{k} \in L_{\mathrm{loc}}^{4}\left(\mathbb{R}^{k}\right)$ with $\nabla \cdot a \in L_{\mathrm{loc}}^{2}\left(\mathbb{R}^{k}\right)$ (distributional derivatives). Then

$$
H=\sum_{j=1}^{k}\left(i \partial_{j}-a_{j}\right)^{2}+V
$$

is essentially self-adjoint on $C_{0}^{\infty}\left(\mathbb{R}^{k}\right)$.

Remarks:

(1) For a proof, see Cycon et al. ${ }^{53}$

(2) This is essentially a best possible result. If $a=0, H$ is defined on $C_{0}^{\infty}$ if and only if $V$ $\in L_{\text {loc }}^{2}$; so the result says for positive $V$, we have essential self-adjointness if and only if $H$ is defined. Similarly, unless there are cancellations, $a_{j} \in L_{\mathrm{loc}}^{4}$ and $\nabla \cdot a \in L_{\mathrm{loc}}^{2}$ is required for $H$ to be defined on $C_{0}^{\infty}$.

(3) It was Simon ${ }^{239}$ who first realized that for $V \geqslant 0$, there only needed to be local $L^{2}$ condi-

tions. However, he required a global condition $\int|V(x)|^{2} e^{-b x^{2}} d x<\infty$ for some $b>0$. It was Kato $^{152}$ who proved the general $a=0$ result (and also allowed for smooth $a$ 's). Kato's paper used the distributional inequality, now called Kato's inequality

$$
\Delta|u| \geqslant \operatorname{Re}(\operatorname{sgn} u \Delta u)
$$

that is also critical to the Leinfelder-Simader proof.

(4) (III.6) is essentially equivalent to the fact that $e^{t \Delta}$ is positivity preserving. The version of (III.6) with magnetic fields is equivalent to diamagnetic inequalities:

$$
\left|\left(e^{-t H} \varphi\right)(x)\right| \leqslant\left(e^{t \Delta}|\varphi|\right)(x)
$$


for the $H$ of (III.5) (with $V \geqslant 0$ ). These ideas were discovered by Nelson, ${ }^{198}$ Simon,,${ }^{241,247}$ and Hess et al. ${ }^{119}$

While there are best possible self-adjointness results for magnetic fields and positive potentials, the results for $V$ 's which can be negative are not in such a definitive form. All the basic principles are understood but I am not aware of a single result that puts them all together (one of the best results is in Kato's paper ${ }^{151}$ although, as we will see, it is not quite optimal with regard to local singularities). So I will present the general principles that are understood in this case.

(a) $-|x|^{2}$ borderline for behavior at infinity. Negative potentials $V$ of compact support for which $H=-\Delta+V$ is essentially self-adjoint on $C_{0}^{\infty}$ normally obey a global estimate of the form (III.3) (with $A=-\Delta, B=V$ ) and, in particular, $H$ is bounded from below. However, if $V$ is not of compact support, it can go to minus infinity at infinity without destroying self-adjointness. More or less, the borderline for keeping self-adjointness is $-|x|^{2}$. For example, it can be proven (see, e.g., Reed and Simon, ${ }^{211}$ Theorem X.9) that $-\left(d^{2} / d x^{2}\right)-|x|^{\alpha}$ on $L^{2}(-\infty, \infty)$ is essentially self-adjoint on $C_{0}^{\infty}(-\infty, \infty)$ if and only if $\alpha \leqslant 2$. This is attractive since a classical particle with the same potential reaches infinity in finite time if and only if $\alpha>2$. Nelson has examples (see Reed and Simon, ${ }^{211}$ p. 156) of $V(x)$ with $V(x) \leqslant-c x^{4}$ so $-\left(d^{2} / d x^{2}\right)+V(x)$ is still essentially self-adjoint and thus, the borderline will not be if and only if, but the general version of this is that if $V(x)$ $\geqslant-c x^{2}$ in some averaged sense, then $-\Delta+V(x)$ will be essentially self-adjoint on $C_{0}^{\infty}$. The earliest version of this is Ikebe and Kato. ${ }^{130}$ My favorite theorem of this genre is due to Faris and Lavine $^{80}$ (see Reed and Simon, ${ }^{211}$ Theorem X.38). In particular, Stark Hamiltonians where $V$ $=\mathbf{c} \cdot \mathbf{x}+V_{0}$ are essentially self-adjoint for suitable $V_{0}$. In any event, I will focus henceforth on cases where $-\Delta+V$ is not unbounded from below.

(b) Stability of relative boundedness under adding $V \geqslant 0$ or a magnetic field. Suppose $A \geqslant 0$. Then (III.3) holds for some $\alpha<1$ if and only if

$$
\lim _{\gamma \rightarrow \infty}\left\|B(A+\gamma)^{-1}\right\|<1 .
$$

On the other hand, (III.7) implies that for $V \geqslant 0$, any a and any multiplication operator $W$ :

$$
\left\|W(H+\gamma)^{-1}\right\| \leqslant\left\|W(-\Delta+\gamma)^{-1}\right\|
$$

and so the second principle is that in studying the negative part of $V$, one can assume $V$ is negative and then add back an arbitrary positive $L_{\text {loc }}^{2}$ positive $V$. While this is true, it ignores situations where there are cancellations between the positive and negative parts which can occur (see, e.g., Combescure and Ginibre ${ }^{48}$ ).

(c) Relative bounds need only hold uniformly locally. The following proposition holds:

Proposition III.3: Suppose $V$ is a function on $\mathbb{R}^{d}$ so that for some $\alpha, \beta$ and every $y$,

$$
\|V \chi(\cdot-y) \varphi\| \leqslant \alpha\|-\Delta \varphi\|+\beta\|\varphi\|
$$

where $\chi$ is the characteristic function of the unit cube. Then for any $\widetilde{\alpha}>\alpha$, there is some $\widetilde{\beta}$ so that

$$
\|V \varphi\| \leqslant \widetilde{\alpha}\|-\Delta \varphi\|+\widetilde{\beta}\|\varphi\| .
$$

This result is proven by a variant of an idea of Sigal. ${ }^{231}$ Find a "partition of unity" $\left\{j_{\mu}\right\}_{\mu}$ so that $\Sigma j_{\mu}^{2}=1$, each $j_{\mu}$ is supported in some unit cube (so $j_{\mu} \chi\left(\cdot-y_{\mu}\right)=j_{\mu}$ for some $j_{\mu}$ ), and the $j_{\mu}$ 's are locally finite, $\Sigma\left(\nabla j_{\mu}\right)^{2}$ is uniformly bounded (the $j_{\mu}$ 's can be translates of a single $j_{\mu}$ ) and $\Sigma\left|\Delta j_{\mu}\right|$ is uniformly bounded. If $H_{0}=-\Delta$, we have (where $C$ is related to $\left\|\Sigma\left(\nabla j_{\mu}\right)^{2}\right\|_{\infty}$ and $\left.\left\|\Sigma\left(\Delta j_{\mu}\right)\right\|_{\infty}\right)$

$$
\sum_{\mu}\left[j_{\mu},\left[j_{\mu}, H_{0}^{2}\right]\right] \leqslant C\left(H_{0}+1\right)
$$

and from this that 


$$
\sum\left\|H_{0} j_{\mu} \varphi\right\|^{2} \leqslant(1+\varepsilon)\left\|H_{0} \varphi\right\|^{2}+C_{\varepsilon}\|\varphi\|^{2} .
$$

Thus

$$
\begin{aligned}
\|V \varphi\|^{2} & =\sum_{\mu}\left\|V \chi\left(\cdot-y_{\mu}\right) j_{\mu} \varphi\right\|^{2} \\
& \leqslant(1+\varepsilon) \alpha^{2} \sum_{\mu}\left\|H_{0} j_{\alpha} \varphi\right\|^{2}+\left(1+\varepsilon^{-1}\right) \beta^{2}\|\varphi\|^{2} \quad \text { (by (III.8) } \\
& \leqslant(1+\varepsilon)^{2} \alpha^{2}\left\|H_{0} \varphi\right\|^{2}+\left(\left(1+\varepsilon^{-1}\right) \beta^{2}+C_{\varepsilon}\right)\|\varphi\|^{2} \quad \text { (by III.10) }
\end{aligned}
$$

which yields (III.9).

Proposition III.3 states that the proper condition on $V$ to yield a $-\Delta$ bound is a uniform local condition.

(d) Convolution results are the proper local condition. As discussed earlier, $L^{p}$ conditions on $V$ do not properly control functions on subspaces. Explicitly, let $\pi: \mathrm{R}^{k} \rightarrow \mathrm{R}^{l}$ be a projection and $V(x)=W(\pi(x))$. Then for $V$ to be $-\Delta$ bounded (assuming $k \geqslant l \geqslant 5$ ), we need $W \in L_{\text {loc }}^{p}\left(\mathbb{R}^{l}\right)$ for $p \geqslant l / 2$ and so $V \in L_{\mathrm{loc}}^{p}\left(\mathrm{R}^{k}\right)$ with $p \geqslant l / 2$. But if $V$ is not a function of a subset of variables, in general we need $p \geqslant k / 2$. It is a discovery of Stummel ${ }^{262}$ that by stating conditions in terms of convolution estimates, one can find conditions that respect subsets of variables. In particular, the following is a space $S_{\nu}$ introduced in Stummel: ${ }^{262}$ Let $V$ be a function on $\mathbb{R}^{\nu}$; we say $V \in S_{\nu}$ if and only if

$$
\begin{gathered}
\lim _{\alpha \downarrow 0}\left[\sup _{x} \int_{|x-y| \leqslant \alpha}|x-y|^{4-\nu}\left|V(y)^{2}\right| d^{\nu} y\right]=0 \quad \text { if } \nu \geqslant 5, \\
\lim _{\alpha \downarrow 0}\left[\sup _{x} \int_{|x-y| \leqslant \alpha} \ln \left(|x-y|^{-1}\right)|V(y)|^{2} d^{\nu} y\right]=0 \quad \text { if } \nu=4 \\
\sup _{x} \int_{|x-y| \leqslant 1}|V(y)|^{2} d^{\nu} y<\infty \quad \text { if } \nu \leqslant 3 .
\end{gathered}
$$

This class respects functions of subvariables in the sense that if $\pi: R^{k} \rightarrow R^{l}$ is a projection, $V(x)=W(\pi(x))$ and $W \in S_{l}$, then $V \in S_{k}$. Moreover, it is not hard to show (see, e.g., Cycon et $a .^{53}$ ) that if $V \in S_{\nu}$, then $V$ is $-\Delta$ bounded with relative bound zero. Moreover (see Cycon et al. ${ }^{53}$ Theorem 1.9), if for some $a, b>0$ and $\delta$ with $0<\delta<1$ and all $0<\varepsilon<1$ and $\varphi \in D\left(H_{0}\right)$

$$
\|V \varphi\|^{2} \leqslant \varepsilon\|\Delta \varphi\|^{2}+a \exp \left(b \varepsilon^{-\delta}\right)\|\varphi\|^{2}
$$

then $V$ is in $S_{\nu}$. See Schechter ${ }^{226}$ for more on Stummel conditions.

(3) The Kato class and going beyond relative boundedness. In his inequality paper, ${ }^{152}$ Kato introduced a form analog $K_{\nu}$ of $S_{\nu}$ : Let $V$ be a function on $\mathbb{R}^{\nu}$; we say $V \in K_{\nu}$ if and only if

$$
\lim _{\alpha \downarrow 0}\left[\sup _{x} \int_{|x-y| \leqslant \alpha}|x-y|^{2-\nu}|V(y)| d^{\nu} y\right]=0 \quad \text { if } \nu \geqslant 3,
$$




$$
\begin{gathered}
\lim _{\alpha \downarrow 0}\left[\sup _{x} \int_{|x-y|<\alpha} \ln \left(|x-y|^{-1}\right)|V(y)| d^{\nu} y\right]=0 \quad \text { if } \nu=2, \\
\sup _{x} \int_{|x-y| \leqslant 1}|V(y)| d^{\nu} y<\infty \quad \text { if } \nu=1 .
\end{gathered}
$$

Then Kato ${ }^{151}$ proved if $\max (-V, 0) \in K_{\nu}$ and $V \in L_{\text {loc }}^{2}(\mathbb{R})$, then $-\Delta+V$ is essentially selfadjoint on $C_{0}^{\infty}\left(\mathbb{R}^{\nu}\right)$. While it is not Kato's proof, this is intimately connected with the semigroup result discussed in Sec. IV. Defining the form sum $H$, one knows $\exp (-t H): L^{2} \rightarrow L^{\infty}$ so $L^{\infty} \cap L^{2} \cap D(H)$ is a domain of essential self-adjointness. It is not hard to then show $L_{0}^{\infty} \cap D(H)$, the $L^{\infty}$ functions of compact support are a domain of essential self-adjointness. Then convolution allows one to get $C_{0}^{\infty}$ approximations.

(f) Logarithmic improvements. Neither $S_{\nu}$ nor $K_{\nu}$ is quite the ideal space for essential selfadjointness. For example, if $\nu \geqslant 5$ and $V(x)=|x|^{-2}(1+|\log | x||)^{-\alpha}, V$ is in $K_{\nu}$ only if $\alpha>1$, in $S_{\nu}$ only if $\alpha>\frac{1}{2}$, but $-\Delta$ bounded with relative bound zero if $\alpha>0$.

Analogous to the issue of self-adjointness is a question of whether maximal and minimal forms agree. This is discussed in Kato ${ }^{152}$ and $\operatorname{Simon}^{248}$ (see Theorem 1.13 in Cycon et al. ${ }^{53}$ ).

\section{PROPERTIES OF EIGENFUNCTIONS, GREEN'S FUNCTIONS, SEMIGROUPS, AND ALL THAT}

I wrote a long review of these subjects 20 years ago $\left(\operatorname{Simon}^{250}\right)$ and the situation has hardly changed since then, although there has been extensive interesting work on what happens for general elliptic operators and for bounded regions (see, e.g., Davies ${ }^{55}$ ). So it will suffice to hit a few major themes. The basic theorem is

Theorem IV.1: Let $V_{+} \in L_{\mathrm{loc}}^{1}\left(\mathbb{R}^{\nu}\right)$ and $V_{-} \in K_{\nu}$, the space of (III.12). Let $H=-\Delta+V$ as a form sum. Then for any $p \leqslant q, e^{-t H}$ maps $L^{p}$ to $L^{q}$ and for $t \leqslant 1$,

$$
\left\|e^{-t H}\right\|_{p, q} \leqslant C t^{-\alpha}
$$

where

$$
\alpha=\frac{\nu}{2}\left(\frac{1}{p}-\frac{1}{q}\right)
$$

Remarks:

(1) Semigroup $L^{p}$ bounds were first found by Davies, ${ }^{54}$ Herbst and Sloan, ${ }^{118}$ and Kovalenko and Semenov ${ }^{161}$ with further developments by Carmona, ${ }^{41}$ Simon, ${ }^{246}$ and Aizenman and Simon. ${ }^{12}$

(2) In particular, it was Aizenman and Simon ${ }^{12}$ who found that $K_{\nu}$ is the natural class for $L^{p}$ bounds. Indeed, they not only proved Theorem IV.1 in this form but also showed that if $V \leqslant 0$ and $\exp (-t H)$ maps $L^{\infty}$ to itself with $\lim _{t \downarrow 0}\left\|e^{-t H}\right\|_{\infty, \infty}=1$, then $V \in K_{\nu}$.

(3) The result holds when magnetic fields are added (by a diamagnetic inequality).

(4) Most of these authors use a combination of path integral estimates and $L^{p}$ interpolation theory. In particular, the Feynman-Kac and Feynman-Kac-Itô formulas (see Simon ${ }^{246}$ for extensive discussion) are useful tools in studying Schrödinger operators. See Simon $^{259}$ for an extension to cases when $V(x) \geqslant-c x^{2}$.

(5) In fact, $e^{-t H}$ takes $L^{p}$ not only into $L^{\infty}$ but into the continuous functions (see Simon, ${ }^{250}$ Theorem B.3.1).

(6) (IV.1)/(IV.2) are precisely the best results for $H=-\Delta$.

(7) This theorem says that $H$ can be defined as the generator of a semigroup on each $L^{p}$ space. The spectrum has been shown to be $L^{p}$ independent in Hempel and Voight. ${ }^{113}$ For a general discussion of $L^{p}$ Schrödinger operators, see Davies. ${ }^{56}$ 
Once one has these estimates, they can be used to derive:

(a) Sobolev estimates: As in the free case if $V$ obeys the conditions of Theorem IV.1, then $(H-z)^{-\nu}$ takes $L^{p}$ to $L^{q}$ if

$$
p^{-1}-q^{-1}<\left(\frac{2 \alpha}{\nu}\right)
$$

The result (see Simon, ${ }^{250}$ Theorem B.2.1) is obtained by integrating the semigroup bound. (IV.3) comes from (IV.2) and the requirement of integrability at $t=0$.

(b) Integral kernels: Bounded operators from $L^{1}$ to $L^{\infty}$ have bounded integral kernels and so Theorem IV.1 can be used (see Simon, ${ }^{250}$ Theorem B.7.1) to prove $e^{-t H},(H-z)^{-\alpha}(\alpha>\nu / 2)$ are integral operators with continuous integral kernels. One can also show (Simon, ${ }^{250}$ Theorem B.7.2) that for $0<\alpha<\nu / 2,(H-z)^{-\alpha}$ is an integral operator with an integral kernel that is continuous away from $x=y$ with a precise singularity at $x=y$.

(c) Eigenfunctions: Since global eigenfunctions (i.e., $\varphi \in L^{2}$ that obey $H \varphi=E \varphi$ ) are in $\operatorname{Ran}\left(e^{-t H}\right)$, Theorem IV.1 implies such eigenfunctions are in $L^{\infty}$. In fact, all this can be done locally. Any eigenfunction (distributional solution of $H \varphi=E \varphi$ ) is automatically continuous and one can prove Harnack inequalities and subsolution estimates. This is discussed in detail in Aizenman and Simon ${ }^{12}$ and Simon. ${ }^{250}$

We end this section with a discussion of some issues involving eigenfunctions. There is much literature on when Schrödinger operators have positive solutions. This was begun by Allegretto ${ }^{13}$ and Piepenbrink ${ }^{206}$ with later results by Agmon $^{5}$ and Pinchover. ${ }^{207}$

Here is a typical theorem (Simon [Ref. 250, Theorem C.8.1]):

Theorem IV.2: Let $V_{-} \in K_{\nu}$ and $K_{+} \in K_{\nu}^{\text {loc }}$. Then $H u=E u$ has a nonzero distributional solution which is everywhere positive if and only if inf $\operatorname{spec}(H) \geqslant E$.

There is also much literature on the issue of exponential decay of eigenfunctions. One result (see Simon ${ }^{250}$, Theorem C.3.1) says that any $L^{2}$ eigenfunction actually goes to zero pointwise-of interest only for eigenfunctions of embedded eigenvalues. For discrete spectrum, the decay is at least exponential under minimal regularity hypothesis on $V$. The original key papers on this theme are by O'Connor ${ }^{200}$ and Combes and Thomas. ${ }^{47}$ From their ideas, one obtains (see Sec. C.3 of Simon); ${ }^{250}$

Theorem IV.3: Let $V_{-} \in K_{\nu}, V_{+} \in K_{\nu}^{\mathrm{loc}}$ and let $H=-\Delta+V$ and let $H u=E u$ with $u \in L^{2}$. Then

$$
|u(x)| \leqslant C e^{-A|x|}
$$

where:

(i) For general $E$ in the discrete spectrum, (IV.4) holds for some $A>0$ and $C>0$.

(ii) If H has compact resolvent, then (IV.4) holds in the sense for any $A>0$, there is a suitable $C>0$.

(iii) If $\Sigma_{\mathrm{ess}}=\inf \sigma_{\mathrm{ess}}(H)$ and $E<\Sigma_{\mathrm{ess}}$, then (IV.4) holds in the sense that for any $A$ $\leqslant \sqrt{E-\Sigma_{\text {ess }}}$, there is a suitable $C>0$.

One can go beyond this to get fairly detailed behavior on decay in cases when $H$ has compact resolvent or for $N$-body potentials. In one dimension, one can justify under some regularity conditions the WKB formula that states when $V(x) \rightarrow \infty$, eigenfunctions decay like

$$
V(x)^{-1 / 4} \exp \left(-\int_{a}^{x} \sqrt{V(y)-E} d y\right) .
$$

It was Agmon ${ }^{4}$ who realized the proper higher-dimensional analog for this involves what is now called the Agmon metric: $\rho(x)$ is the geodesic distance of $x$ to 0 in the Riemannian metric 
$\rho_{i j}(x)=\delta_{i j}(V(x)-E)_{+} d^{2} x$. There is a related but more subtle definition for $N$-body systems. See Agmon $^{4}$ and Deift et al. ${ }^{59}$ for further discussions. See Simon ${ }^{253}$ and Helffer and Sjöstrand ${ }^{111}$ for an application to tunneling probabilities.

Eigenfunctions play a critical role in explicit spectral representations of Schrödinger operators. The basic ideas go back to work of Browder, ${ }^{39}$ Garding, ${ }^{88} \mathrm{Gel}$ 'fand, ${ }^{89} \mathrm{Kac},{ }^{137}$ and especially Berezanskii. ${ }^{29,30}$ See Sec. C.5 of $\operatorname{Simon}^{250}$ and Last and $\operatorname{Simon}^{170}$ for some additional onedimensional results.

Finally, we mention issues of cusps and nodes of eigenfunctions. Kato ${ }^{148}$ has a famous paper on cusps at Coulomb singularities for atomic eigenfunctions. See Hoffmann-Ostenhof et al. ${ }^{108,120,121}$ for recent developments in this area.

\section{ONE-DIMENSIONAL DECAYING POTENTIALS}

One-dimensional Schrödinger operators

$$
-\frac{d^{2}}{d x^{2}}+V(x)
$$

on $L^{2}(-\infty, \infty)$ and $L^{2}(0, \infty)$ and their discrete analogs

$$
h u(n)=u(n+1)+u(n-1)+V(u) u(n)
$$

on $l^{2}(-\infty, \infty)$ and $l^{2}[0, \infty)$ have been heavily studied for two reasons. First, ordinary differential equation (ODE)/difference equation methods allow one to study them in much greater detail than one can the higher-dimensional analogs. Second, if $V(x)=V(|x|)$ is a spherically symmetric function on $\mathbb{R}^{\nu}$, then $-\Delta+V$ is unitarily equivalent to a direct sum of operators on $L^{2}(0, \infty)$ or the form (V.1) where the effective $V$ 's have the form $V_{l}(x)=\kappa_{l}|x|^{-2}+V(x)$ for suitable $\kappa_{l}$ 's. The details can be found, for example, in Reed and Simon, ${ }^{211}$ Example 4 to the Appendix for X.1.

The one-dimensional theory has been in and out of vogue. It was extensively studied from 1930 to 1950 with important contributions by Titchmarsh, Kodaira, Gel'fand, Hartman-Wintner, Levinson, Coddington-Levinson, and Jost. Significant developments during the next 25 years were mainly in the area of inverse spectral theory (a major exception was Weidmann's work, ${ }^{273}$ to be discussed shortly) which will be discussed in Sec. VI. From about 1975 starting with the work of Goldsheid et al. ${ }^{98}$ and Pearson, ${ }^{204}$ this has been an active area with extensive study of the one-dimensional case, especially with long-range and with ergodic potentials.

One special feature of one dimension is that one can limit spectral multiplicities under very general conditions on $V$ :

\section{Theorem V.1:}

(a) Let $H=-\left(d^{2} / d x^{2}\right)+V(x)$ on $L^{2}(0, \infty)$ with fixed $h u(0)+u^{\prime}(0)=0$ boundary conditions and suppose $H$ is essentially self-adjoint on $C_{0}^{\infty}[0, \infty)$. Then $H$ has simple spectrum (multiplicity 1).

(b) Let $H=-\left(d^{2} / d x^{2}\right)+V(x)$ on $L^{2}(-\infty, \infty)$ and suppose $H$ is essentially self-adjoint on $C_{0}^{\infty}(-\infty, \infty)$. Then

(i) The absolutely continuous spectrum of $H$ is of multiplicity at most 2.

(ii) The singular spectrum of $H$ is of multiplicity 1.

Remarks:

(1) All one needs for local regularity of $V$ is $V \in L^{1}[0, R]$ for all $R>0$ or $L_{\text {loc }}^{1}(-\infty, \infty)$.

(2) The result holds even if $H$ is not essentially self-adjoint ( $V$ limit circle at $\pm \infty$ ) so long as a boundary condition is imposed at $\infty$ or at $-\infty$.

(3) The only subtle part of the result is that the singular continuous spectrum is simple on the real line. This is a theorem of Kac; ${ }^{138,139}$ see also Berezanskii. ${ }^{29,30}$ My preferred proof is due to Gilbert. ${ }^{95,96}$ 
In this section, we will discuss the case where $V(x) \rightarrow 0$ at infinity. In Sec. VI, we will discuss inverse spectral theory, and in Sec. VII, we will discuss ergodic potentials. (These two subjects are mainly one dimensional.) The issue of the asymptotic eigenvalue distribution when $V \rightarrow \infty$ as $\pm \infty$ is discussed in Sec. XIV on the quasiclassical limit.

This section will discuss (V.1)/(V.2) in situations where $V(x)$ (or $V(n)$ ) goes to zero (at least in an average sense) as $x \rightarrow \infty$ (or $n \rightarrow \infty)$. The interesting thing is that there are three natural breaks in behavior. Expressed in terms of $|x|^{-\alpha}$ behavior, they are

(i) At $\alpha=2$, we shift between a finite number of bound states $(\alpha>2)$ or an infinite number $(\alpha<2)$ at least if $V(x)<0$.

(ii) At $\alpha=1\left(V \in L^{1}\right)$, we shift between a pure scattering situation for positive energies $(\alpha$ $>1)$ and the possibility of positive energy bound states $(\alpha<1)$.

(iii) At $\alpha=\frac{1}{2},\left(V \in L^{2}\right)$, we shift from there being a.c. spectrum for almost everywhere positive energy $\left(\alpha>\frac{1}{2}\right)$ to at least the possibility of very different spectrum.

(i) and (ii) have been known since the earliest days of quantum mechanics. The $\alpha=\frac{1}{2}$ borderline first occurred in Simon ${ }^{251}$ who found that random decay potentials had point spectrum when $\alpha<\frac{1}{2}$. Delyon et al. ${ }^{64}$ then showed if $\alpha=\frac{1}{2}$, there may be some nonpoint spectrum. As we will see, subsequent results confirmed this borderline.

The negative spectrum for decaying potentials is easy: So long as $\int_{x}^{x+1}|V(y)| d y \rightarrow 0, H$ is bounded below and has $[0, \infty)$ as essential spectrum by Weyl's criterion (see, e.g., Reed and Simon, ${ }^{213}$ Sec. XIII.4), which means that $(-\infty, 0)$ has only discrete eigenvalues of finite multiplicity, which can only accumulate at energy 0 . Indeed, by Theorem V.1, the point spectrum is of multiplicity 1 . Once these basics are established for the discrete spectrum, a number of detailed questions about it arise:

(a) Is $\sigma_{\text {disc }}$ finite or infinite? The borderline, as mentioned above, is $r^{-2}$ decay. Explicitly, one has Bargmann's bound ${ }^{24}$ that the number of eigenvalues on a half line with $u(0)=0$ boundary conditions is bounded by $\int x|V(x)| d x$ and on a whole line by $1+\int_{-\infty}^{\infty}|x||V(x)| d x$ (see $\operatorname{Simon}^{240}$ for a review of bounds on the number of bound states). On the other hand, if $\overline{\lim }_{x \rightarrow \infty}|x|^{2} V(x)$ $\leqslant-\frac{1}{4}$, one can prove that $H$ has an infinity of bound states (see, e.g., Reed and Simon, ${ }^{213}$ Theorem XIII.6).

(b) If $\sigma_{\text {disc }}$ is infinite, how does $\lim _{\lambda \uparrow 0} \operatorname{dim} E_{(-\infty, \lambda)}(H)$ diverge? This is a quasiclassical limit and discussed in Sec. XIV.

(c) Bounds on moments of eigenvalues. Lieb and Thirring, ${ }^{186}$ motivated in part by their work on the stability of matter, ${ }^{185}$ initiated extensive study on the best constant $L_{\gamma, 1}$ in

$$
\sum_{j}\left|e_{j}\right|^{\gamma} \leqslant L_{\gamma, 1} \int|V(x)|^{\gamma+1 / 2} d x,
$$

which holds if $\gamma \geqslant \frac{1}{2}$. Here $\left\{e_{j}\right\}$ are the negative eigenvalues of $H$. For $\gamma \geqslant \frac{3}{2}$, the constant $L_{\gamma, 1}$ is known to be quasiclassical (Aizenman and Lieb). ${ }^{9}$ For $\gamma \in\left[\frac{1}{2}, \frac{3}{2}\right.$ ), it is known that $L_{\gamma, 1}$ is strictly larger than the quasiclassical result. ${ }^{186}$ It is conjectured to be the optimal value for a single bound state, as explained in Lieb and Thirring, ${ }^{186}$ but this is still open (except at $\gamma=\frac{1}{2}$ (Hundertmark et al. $\left.^{125}\right)$.

(d) Is there a bound state for weak coupling? In one (and two) dimensions, $H$ has bound states even for very weak coupling. The result $\left(\operatorname{Simon}^{242}\right)$ is that if $\int|x||V(x)| d x<\infty$ and $\int V(x) d x$ $\leqslant 0$ and $V \neq 0$, then $H$ always has a bound state and the binding energy of $-\Delta+\mu V$ is $\sim c \mu^{2}$ as $\mu \downarrow 0$ (if $\int V(x) d x<0$; it is $\sim c \mu^{4}$ if $\int V(x)=0$ ).

As for positive energies, the situation is simple if $V \in L^{1}$ :

Theorem V.2: Let $V \in L^{1}(-\infty, \infty)$ or $L^{1}(0, \infty)$. Then $H E_{(0, \infty)}(H)$ is unitarily equivalent to $-d^{2} / d x^{2}\left(\right.$ on $L^{2}(-\infty, \infty)$ or $L^{2}(0, \infty)$ with $u(0)=0$ boundary conditions).

Remarks:

(1) This result is essentially due to Titchmarsh. ${ }^{267}$

(2) In terms of $r^{-\alpha}$ falloff, $V \in L^{1}$ means $\alpha>1$. 
(3) Using scattering theoretic ideas, one can prove wave operators exist and are complete (see Sec. VIII).

(4) This says there is no point of singular continuous spectrum at positive energies and that the a.c. spectrum has essential support $(0, \infty)$ with multiplicity 2 or 1 .

(5) We have stated the result for $u(0)=0$ boundary condition for simplicity; it holds for all boundary conditions at 0 .

As for slower decay than $L^{1}$, if one has control of derivatives, one can still conclude the positive spectrum is purely absolutely continuous. The simplest result of this genre is

Theorem V.3: $\left(\right.$ Weidmann ${ }^{273}$ ) Let $V=V_{1}+V_{2}$ where $V_{1}$ is in $L^{1}, V_{2}(x) \rightarrow 0$ as $x \rightarrow \pm \infty$, and $V_{2}$ is of bounded variation. Then, $H E_{(0, \infty)}(H)$ is unitarily equivalent to $-d^{2} / d x^{2}$ (on $L^{2}$ $(-\infty, \infty)$ or on $L^{2}(0, \infty)$ with $u(0)=0$ boundary conditions $)$.

Remarks:

(1) $V_{2}$ of bounded variation with $V_{2} \rightarrow 0$ at infinity essentially says that $-d V_{2} / d x \in L^{1}$; in fact, any $V_{2}$ of bounded variation can be written $V_{3}+V_{4}$ with $V_{3} \in L^{1}$ and $V_{4}$ a $C^{1}$ function with $d V_{4} / d x \in L^{1}$.

(2) Pure power potentials $r^{-\alpha}$ for any $\alpha>0$ are included in this theorem; indeed, any monotone function $V(x)$ with $V(x) \rightarrow 0$ as $x \rightarrow \infty$ is of bounded variation.

For a short proof of Theorems V.2/V.3, see Simon. ${ }^{256}$ Both theorems can be understood as coming from the fact that all solutions of $-u^{\prime \prime}+V u=\lambda u$ with $\lambda>0$ are bounded. That such a conclusion implies the spectrum is purely absolutely continuous was first indicated by Carmona ${ }^{42}$ (who required some kind of uniformity in $\lambda$ ). Important later developments that capture this idea are due to Gilbert and Pearson, ${ }^{97}$ Last and Simon, ${ }^{170}$ and Jitomirskaya and Last. ${ }^{135}$ The tools in those papers are also important for the proofs of the results of Sec. VII.

Once one allows decay slower than $r^{-1-\epsilon}$ for both $V$ and $V^{\prime}$, the conclusion of Theorems V.2/V.3 can fail because of embedded point spectrum. The original examples of this were found by von Neumann and Wigner. ${ }^{272}$ Basically, if $V(x)=\gamma|x|^{-1} \sin (x)$ for $x$ large and $\gamma>1$, then $-u^{\prime \prime}+V u=\frac{1}{4} u$ has a solution which is $L^{2}$ at infinity (see, e.g., Theorem XI.67 in Reed and Simon $^{213}$ ). By adjusting $V$ at finite $x$, one can arrange for any boundary condition one wants at $x=0$. In fact, if one allows slightly slower decay than $|x|^{-1}$, one can arrange dense point spectrum. Naboko ${ }^{197}$ and Simon ${ }^{257}$ have shown that for any sequence $\left\{\lambda_{n}\right\}_{n=1}^{\infty}$ of energies in $(0, \infty)$ (Naboko has a mild restriction on the $\lambda$ 's) and any $g(r)$ obeying $\lim _{r \rightarrow \infty} r g(r)=\infty$, there is a $V(x)$ obeying:

(i) $\quad|V(x)| \leqslant g(|x|)$ for $x$ large;

(ii) $\quad-u^{\prime \prime}+V u=\lambda_{n} u$ has a solution $L^{2}$ at infinity and obeying a prescribed boundary condition at $x=0$.

Remark: It is an interesting open question about whether there exist potentials decaying faster than $|x|^{-1 / 2-\varepsilon}$ with dense singular continuous spectrum (rather than dense point spectrum).

The interesting fact is that even though potentials of Naboko-Simon type have dense point spectrum, they may also have lots of a.c. spectrum. The best result is:

Theorem V.4: (Deift and Killip ${ }^{60}$ ) Let $V \in L^{2}$. Then the essential support of the a.c. spectrum of $H=-\left(d^{2} / d x^{2}\right)+V$ is $[0, \infty)$.

Remarks:

(1) In terms of $r^{-\alpha}$ decay, this result requires $\alpha>\frac{1}{2}$.

(2) This result is optimal in that it is known for any Orlicz space strictly larger than $L^{2}$ in terms of behavior at infinity, there are $V$ 's whose associated $H$ has no a.c. spectrum.

(3) The first result of this genre was found by Kiselev ${ }^{156}$ who proved the conclusion of this theorem for $|V(x)| \leqslant C x^{-3 / 4-\epsilon}$. There were subsequent improvements of this by Kiselev, ${ }^{157} \mathrm{Christ}$ and Kiselev, ${ }^{46}$ and Remling. ${ }^{218}$

(4) Killip ${ }^{155}$ has a partially alternate proof of Theorem V.4. 

$[0, \infty)$ :

Once the decay is allowed to be slower than $r^{-1 / 2}$, one can have much different spectrum in

(i) If $W$ is a suitable family of random homogeneous potentials and $V(x)=|x|^{-\alpha} W(x)$ with $\alpha<\frac{1}{2}$, then $H$ has only dense point spectrum in $(0, \infty)$. This was first proven in the discrete case by Simon $^{251}$ and later in the continuum case by Kotani and Ushiroya. ${ }^{160}$

(ii) Generic potentials decaying like $|x|^{-\alpha}\left(\frac{1}{2}>\alpha>0\right)$ produce singular continuous spectrum as discovered by Simon. ${ }^{255}$ For example, in $\left\{\left.V \in \mathbb{C}(\mathbb{R})\left|\sup _{x}\right| x\right|^{\alpha}|V(x)| \equiv\|V\|_{\alpha}\right\}$ viewed as a complete metric space in $\|\cdot\|_{\alpha}$, a dense $G_{\delta}$ of $V$ 's are such that $-d^{2} / d x^{2}+V(x)$ has purely singular continuous spectrum on $[0, \infty)$.

(iii) Much more is known in the borderline $\alpha=\frac{1}{2}$ case, at least for the discrete Schrödinger operator (V.2). For example, if $a_{n}$ are independent, identically distributed random variables uniformly distributed in $[-1,1]$ and $V(n)=\mu n^{-1 / 2} a_{n}$, then for suitable coupling constants $\mu$ and energies $E$ in $[-2,2]$, the spectral measures have fractional Hausdorff dimension with an exactly computable local dimension. This is discussed in Kiselev et al. ${ }^{158}$ There are earlier results on this model by Delyon et al. ${ }^{64}$ and Delyon. ${ }^{62}$

(iv) A very different class of decaying potentials was studied by Pearson. ${ }^{204}$ His potentials are of the form

$$
V(x)=\sum_{n=1}^{\infty} a_{n} W\left(x-x_{n}\right)
$$

where $W \geqslant 0, a_{n} \rightarrow 0$, and $x_{n} \rightarrow \infty$ very rapidly so the bumps are sparse. He showed that for suitable $a_{n}, x_{n}$, the corresponding $H$ has purely singular spectrum-providing the first explicit examples of such spectrum. Strong versions of his results were found by Remling ${ }^{217}$ and Kiselev et al. ${ }^{158}$ In particular, the latter authors proved if $\left(x_{n+1} / x_{n}\right) \rightarrow \infty$ (e.g., $\left.x_{n}=n !\right)$, then potentials of the form (V.3) lead to $H$ 's with purely singular spectrum if $\Sigma a_{n}^{2}=\infty$ and to ones with purely a.c. spectrum if $\sum a_{n}^{2}<\infty$.

\section{INVERSE SPECTRAL THEORY}

One area related to Schrödinger operators, especially in one dimension, is the question of inverse theory: How does one go from spectral or scattering information to the potential. There is much literature, including three books I would like to refer the reader to: Chadan and Sabatier, ${ }^{45}$ Levitan, ${ }^{176}$ and Marchenko. ${ }^{190}$ I will only touch some noteworthy ideas here.

In one dimension, a key role is played by the Weyl $m$ function and the associated spectral measure $d \rho$. Given a potential $V$ so that $H$ is self-adjoint with $u(0)=0$ boundary conditions, for each $z$ with $\operatorname{Im} z>0$, there is a solution $u(x ; z)$ of $-u^{\prime \prime}+V u=z u$ which is $L^{2}$ at infinity. The $m$ function is defined by

$$
m(z)=\frac{u^{\prime}(0 ; z)}{u(0, z)}
$$

$\operatorname{Im} m(z)>0$ in $\operatorname{Im} z>0$ so by the Herglotz representation theorem

$$
m(z)=B+\int d \rho(\lambda)\left[\frac{1}{\lambda-z}-\frac{\lambda}{1+\lambda^{2}}\right]
$$

for a suitable constant $B . d \rho$ is called the spectral measure for $H$. One can recover $d \rho$ from $m$ by

$$
\frac{1}{\pi} \operatorname{Im} m(\lambda+i \varepsilon) d \lambda \rightarrow d \rho(\lambda)
$$

weakly as $\varepsilon \downarrow 0$ and (VI.2) allows the recovery of $m$ from $d \rho$ given the known asymptotics (Atkinson, ${ }^{15}$ Gesztesy and Simon ${ }^{93}$ ) 


$$
m\left(-\kappa^{2}\right)=-\kappa+o(1)
$$

as $|\kappa| \rightarrow \infty$ with $\delta<\operatorname{Arg} \kappa<(\pi / 2)-\delta$. $d \rho$ really is a spectral measure for let $\widetilde{\varphi}(x, \lambda)$ solve $-\widetilde{\varphi}^{\prime \prime}$ $+V \widetilde{\varphi}=\lambda \widetilde{\varphi}$ with boundary conditions $\widetilde{\varphi}(0, \lambda)=0, \widetilde{\varphi}^{\prime}(0, \lambda)=1$, and define for $f \in C_{0}^{\infty}(0, \infty)$

$$
(U f)(\lambda)=\int \widetilde{\varphi}(x, \lambda) f(x) d x
$$

Then $U$ is a unitary map of $L^{2}(0, \infty, d x)$ to $L^{2}(\mathrm{R}, d \rho(\lambda))$; in particular,

$$
\int|(U f)(\lambda)|^{2} d \rho(\lambda)=\int|f(x)|^{2} d x
$$

or formally

$$
\int \varphi(x, \lambda) \varphi(y, \lambda) d \rho(\lambda)=\delta(x-y)
$$

Moreover, $(U H f)(\lambda)=\lambda(U f)(\lambda) . d \rho$ and its equivalent function $m$ is therefore close to spectral information. One way of seeing this explicitly is if $V(x) \rightarrow \infty$. In that case, $m$ is meromorphic, the poles of $m$ are precisely the eigenvalues of $H$ with $u(0)=0$ boundary conditions and by definition of $m$, the zeros are precisely the eigenvalues with $u^{\prime}(0)=0$ boundary conditions. $m$ is uniquely determined by these two sets of eigenvalues.

In many ways, the fundamental result in inverse theory is the following one:

Theorem VI.1: Borg $^{37}$-Marchenko ${ }^{188}$ ) $m$ determines $q$, that is, if $q_{1}$ and $q_{2}$ have equal m's, then $q_{1}=q_{2}$.

Recently, the following local version of the Borg-Marchenko theorem was proven

Theorem VI.2: Let $q_{1}$ and $q_{2}$ be potentials and $m_{1}$ and $m_{2}$ their $m$ functions. Then $q_{1}=q_{2}$ on $[0, a]$ if and only if

$$
\left|m_{1}\left(-\kappa^{2}\right)-m_{2}\left(-\kappa^{2}\right)\right|=O\left(e^{-2 a \kappa}\right)
$$

as $\kappa \rightarrow \infty$ for $\kappa$ obeying $\delta \leqslant \arg \kappa \leqslant \pi / 2-\delta$.

Remarks:

(1) This result was first proven by Simon ${ }^{258}$ when $q_{1}$ and $q_{2}$ are bounded from below.

(2) The general result which even allows $q_{i}$ to be limit circle at infinity was first obtained by Gesztesy and Simon. ${ }^{93}$

(3) A simple proof of Theorem VI.2 was subsequently obtained by Gesztesy and Simon. ${ }^{94}$

Given the uniqueness result, it is natural to ask about concrete methods of determining $q$ given $m$. There are two approaches for the general case. The first is due to Gel'fand and Levitan ${ }^{90}$ and depends on the orthogonality relation (VI.7), while the other, due to Simon, ${ }^{258}$ is a kind of continuum analog of the continued fraction approach to solving the moment problem.

The Gel'fand-Levitan approach depends on a representation of the solutions $\varphi$ due to Povzner $^{208}$ and Levitan: ${ }^{175}$

$$
\varphi(x, \lambda)=\frac{\sin (k x)}{k}+\int_{0}^{x} K(x, y) \frac{\sin (k y)}{k} d y,
$$

where $\lambda=k^{2}$. In essence, (VI.7) leads to a linear Volterra integral equation for $K$ whose kernel is determined by $\rho$. Once one has $K$, one can determine $V$ from (VI.8) and $-\varphi^{\prime \prime}+V \varphi=\lambda \varphi$ or from more direct relations of $K$ to $V$.

The approach of Simon depends on a representation of $m$ as a Laplace transform 


$$
m\left(-\kappa^{2}\right)=-\kappa-\int_{0}^{a} A(\lambda) e^{-2 \kappa a} d \alpha+O\left(e^{-2 a \kappa}\right),
$$

which determines $A$ given $m$ (there is also a direct relation of $A$ to $\rho$ given in Gesztesy and Simon $\left.^{93}\right)$. One can introduce a second variable and function $A(x, \alpha)$ so $A(x=0, \alpha) \equiv A(\alpha)$. $A$ obeys

$$
\frac{\partial A}{\partial x}=\frac{\partial A}{\partial \alpha}+\int_{0}^{\beta} A(x, \beta) A(x, \alpha-\beta) d \beta
$$

and

$$
\lim _{\alpha \downarrow 0} A(x, \alpha) \equiv V(x)
$$

In this approach, $m$ determines $A(x=0, \cdot)$ by (VI.9); the differential equation (VI.10) determines $A(x, \alpha)$, and then (VI.11) determines $V$.

Inverse spectral theory is connected to inverse scattering for short-range potentials since $d \rho$ on $[0, \infty)$ is determined by scattering data. Scattering data also determine the positions of the negative eigenvalues. One needs to supplement that with the weight of the pure points at these negative eigenvalues known as norming constants. Marchenko ${ }^{190,189}$ has an approach to inverse scattering related to the Gel'fand-Levitan approach by using a different representation than (VI.8). When $\int_{0}^{\infty} x|V(x)| d x<\infty$, Levin ${ }^{174}$ has proven that in $\operatorname{Im} k>0$, there is a solution $\psi(x, k)$ of $-\psi^{\prime \prime}+V \psi=k^{2} \psi$ given by

$$
\psi(x, k)=e^{i x k}+\int_{x}^{\infty} \widetilde{K}(x, y) e^{i k y} d y
$$

Krein ${ }^{162-164}$ also developed an approach to inverse problems. A different approach to inverse scattering is due to Deift and Trubowitz. ${ }^{61}$ For another approach to inverse problems, see Melin. ${ }^{195}$ Inverse theory for periodic potentials also has an extensive literature starting with Dubrovin et al. ${ }^{70}$ Its and Matveev, ${ }^{132}$ McKean and van Moerbeke, ${ }^{193}$ McKean and Trubowitz, ${ }^{192}$ and Trubowitz. ${ }^{268}$

As for higher-dimensional inverse scattering, these scattering data overdetermine the potential. For example, for short-range $V$ 's, the scattering amplitude at fixed momentum transfer approaches the Fourier transform of $V$ at large energy, so the large energy asymptotics of scattering determine $V$. There is considerable literature on recovering $V$ from partial scattering data, which we will not try to summarize here.

One reason for the interest in inverse theory is the connection it sets up between spectral theory of Schrödinger operators and the analysis of certain nonlinear partial differential equations like KdV (see Dodd et al., ${ }^{68}$ Novikov et al., ${ }^{199}$ and Belokolos et al. ${ }^{26}$ ).

\section{ERGODIC POTENTIALS}

Let $\Omega$ be a compact metric space with probability measure $d \gamma$ and $T_{t}$ with $t \in R^{\nu}$ or $T_{n}$ with $n \in \mathbb{Z}^{\nu}$ be an ergodic family of measure-preserving transformations. Let $f: \Omega \rightarrow \mathbb{R}$ be continuous. For $\omega \in \Omega$, define

$$
V_{\omega}(x)=f\left(T_{x} \omega\right)
$$

and

$$
H_{\omega}=-\Delta+V_{\omega} .
$$


Note: To allow unbounded V's as seen, for example, in Gaussian random potentials, one wants to extend this picture to either allow $f$ to be discontinuous and/or take values in $R \cup\{\infty\}$, and/or allow $\Omega$ to be noncompact; for simplicity, we will discuss this model for motivation.

$H_{\omega}$ is a family of Schrödinger operators, not a single one, but by the ergodicity and an obvious translation covariance $V_{T_{v} \omega}(x)=V_{\omega}(x+y)$, many spectral properties occur with the probability one. So one can speak of typical properties. In particular, it is known that the full spectrum $\Sigma$, the essential support of the absolutely continuous spectrum $\Sigma_{\mathrm{ac}}$, the closure of the point spectrum $\Sigma_{\mathrm{pp}}$, and the singular continuous spectrum $\Sigma_{\mathrm{sc}}$ are a.e. constant in $\psi$ (see, e.g., Theorems 9.2 and 9.4 in Cycon et al. ${ }^{53}$ for proofs; the result for $\Sigma$ and $\Sigma_{\text {pp }}$ is due to Pastur ${ }^{202}$ and the other results to Kunz and Souillard ${ }^{165}$ ). Note only $\Sigma_{\mathrm{pp}}$ is a.e. constant; $\Sigma_{\mathrm{pp}}$, the actual set of eigenvalues is not.

Examples:

(1) Let $\Omega=[a, b]^{\mathrm{Z}^{v}}$ and let $d \gamma$ be the infinite product of normalized Lebesgue measure on $[a, b]$. Let $\left(T_{m} \omega\right)_{n}=\omega_{n+m}$. The corresponding discrete Schrödinger operator is called the Anderson model and is typical of random potential models.

(2) If $\Omega$ is a compact Abelian group with $\mathbb{Z}^{\nu}$ or $\mathbb{R}^{\nu}$ as a dense subgroup, $d \gamma$ is the Haar measure and $T_{x}$ is the group translate, then $V$ is a periodic or almost periodic function. A frequently discussed example is

$$
V(n)=\lambda \cos (\pi \alpha n+\theta),
$$

where $\alpha$ is irrational, $\theta$ runs in $[0,2 \pi]$ (which is $\Omega$ ), and $\lambda$ is a parameter. The corresponding discrete Schrödinger operator is called the almost Mathieu model.

The simplest example of this framework-which is atypical in many ways-is the periodic potential. The basic facts in this case go back to physics literature at the start of quantum mechanics (Bloch, Brillouin, Kramer, and Wigner) and, in one dimension, to work on Hill's equation (Floquet, Lyapunov, Hamel, and Haupt). A critical early mathematical paper on the multidimensional case is Gel'fand. ${ }^{89}$ The key result is that for periodic $V$ 's with a mild local regularity condition, $H=-\Delta+V$ has purely absolutely continuous spectrum. This result is discussed in detail in Reed and Simon, ${ }^{212}$ Sec. XIII.16). The only subtle part of the argument is to eliminate the possibility of what are called flatbands, a result of Thomas. ${ }^{264}$

In the mathematical physics literature, the period from 1975 onwards has seen enormous interest in the study of almost periodic and random models and special cases thereof. Three books that discuss this are part of Carmona and Lacroix ${ }^{44}$ Cycon et al.,${ }^{53}$ and Pastur and Figotin. ${ }^{203} \mathrm{We}$ will only touch some of the general principles, leaving the details-especially of detailed models - to the books and the vast literature. We will make references to the Lyapunov exponent without defining it; see Cycon et al., ${ }^{53}$ Sec. 9.3.

For random potentials, the most interesting results concern localization. While the spectrum is typically an interval (e.g., for the Anderson model in $\nu$ dimensions, it is $[a-2 \nu, b+2 \nu]$ ), the spectrum is pure point with eigenvalues dense in the interval and exponentially decaying eigenfunctions.

In one dimension, localization was first rigorously proven by Goldsheid et al. ${ }^{98}$ with a later alternative by Kunz and Souillard. ${ }^{165}$ Following an idea of Kotani, ${ }^{159}$ Simon and Wolff, ${ }^{261}$ and Delyon et al. ${ }^{63}$ found another proof. Typical is

Theorem VII.1: For the one-dimensional Anderson model, the spectrum is $[a-2, b+2]$ and is pure point with probability one with eigenfunctions decaying at the Lyapunov rate.

Carmona et $a l .{ }^{43}$ and Shubin et al. ${ }^{228}$ have approaches that work if the single site distribution is discrete (the other quoted approaches require an absolutely continuous component for this distribution).

In higher dimensions, the two main approaches to localization are due to Fröhlich and Spencer $^{87}$ (see also von Dreifus and Klein ${ }^{270}$ ) and to Aizenman and Molchanov. ${ }^{10}$ (See also Aizenman and $\mathrm{Graf}^{8}$ and Aizenman et al. ${ }^{11}$ ) Basically, these authors and the many papers that extend their ideas prove dense point spectrum in regimes where the coupling constant is large or 
one is near the edge of the spectrum. It is believed—but not proven - that in suitable regimes when $\nu \geqslant 3$, there is absolutely continuous spectrum.

For almost periodic models, one can have any kind of spectral type. The almost Mathieu model has been almost entirely analyzed and the spectral type shows a great variety. Recall this is the discrete model with potential

$$
V_{\alpha, \lambda, \theta}(n)=\lambda \cos (\pi \alpha n+\theta),
$$

where $\lambda, \alpha$ are fixed parameters and $\theta$ runs through $\Omega$. Then

(i) If $\lambda<2$, there is always (i.e., for any irrational $\alpha$ ) lots of a.c. spectrum and it is known for some $\alpha$ and believed for all $\alpha$ that is all there is (see Last, ${ }^{169}$ Gesztesy and Simon, ${ }^{92}$ Gordon et al. ${ }^{100}$ Jitomirskaya ${ }^{134}$ the earliest results of this genre are due to Dinaburg and Sinai ${ }^{67}$ ).

(ii) If $\lambda=2$ and $\alpha$ is an irrational whose continued fraction integers are unbounded (almost all $\alpha$ have this property), then the spectrum is known to be purely singular continuous for almost all $\theta$ (see Gordon et al. ${ }^{100}$ ).

(iii) If $\lambda>2$ and $\alpha$ is an irrational with good Diophantine properties $\left(|\alpha-p / q| \geqslant C q^{-l}\right.$ for some $C, l$ and all $p, q, \in \mathbb{Z}$ ), then for a.e. $\theta$, the spectrum is dense pure point (Jitomirskaya; ${ }^{134}$ see also Bourgain and Goldstein ${ }^{38}$ ).

(iv) If $\lambda>2$ and $\alpha$ is irrational, there are always lots of $\theta$ (a dense $G_{\delta}$ ) for which the spectrum is purely singular continuous (Jitomirskaya and $\operatorname{Simon}^{136}$ ). For some $\alpha$, like those in (iii), the set while a dense $G_{\delta}$ has measure 0 . For Liouville $\alpha$ (irrational $\alpha$ 's with $\lim (1 / q) \ln |\sin \pi \alpha q|=-\infty$ ), the spectrum is purely singular continuous (Avron and Simon ${ }^{22}$ using $\overline{\text { results of Gordon }}{ }^{99}$ ).

In general, for almost periodic models, the spectral type is dependent on the number theoretic properties of the frequencies. Among the general spectral results known for almost periodic models is that the spectrum is everywhere constant on $\Omega$ (rather than only almost everywhere constant; Avron and Simon ${ }^{22}$ ) and that the essential support of the a.c. spectrum is everywhere constant (Last and Simon ${ }^{170}$ ). It is known [see (iv)] that $\bar{\sigma}_{\mathrm{pp}}$ and $\sigma_{\mathrm{sc}}$ may only be almost everywhere constant and fail to be constant on all of $\Omega$.

\section{TWO-BODY HAMILTONIANS}

Hamiltonians of the form $-\Delta+V$ where $V(x) \rightarrow 0$ at infinity are often referred to as two-body Hamiltonians since the Hamiltonian of two particles with a potential $W\left(\mathbf{r}_{1}-\mathbf{r}_{2}\right)$ reduces to $-\Delta$ $+V$ (where $V$ is a multiple of $W$ depending on the masses) after removal of the center of mass. The issues are essentially the same as for one-dimensional decaying potentials as discussed in Sec. V.

With regard to the negative spectrum, again Weyl's criterion easily shows that $\sigma_{\text {ess }}(H)$ $=[0, \infty)$ so that $H$ has only discrete spectrum of finite multiplicity in $(-\infty, 0)$ and only 0 can be an accumulation point. Typical is:

Theorem VIII.1: For $\alpha \in \mathbb{Z}^{\nu}$, let $\chi_{\alpha}$ be the characteristic function of the unit cube about $\alpha$. Let $V: \mathbb{R} \rightarrow R$. Suppose $V \in K_{\nu}$ and that as $\alpha \rightarrow \infty,\left\|\chi_{\alpha} V\right\|_{K_{\nu}} \rightarrow 0$. Then $\sigma_{\text {ess }}(-\Delta+V)=[0, \infty)$.

As for whether $N(V)$, the number of negative bound states (counting multiplicity, i.e., $\left.N(V)=\operatorname{dim} E_{(-\infty, 0)}(H)\right)$, is finite or infinite, there is considerable literature. The earliest bound is due to Birman ${ }^{32}$ and Schwinger ${ }^{227}$ for $\nu=3$. It states

$$
N(V) \leqslant \frac{1}{(4 \pi)^{2}} \int \frac{|V(x)||V(y)|}{|x-y|^{2}} d x d y \quad(\nu=3) .
$$

Perhaps the most famous bound is that of Cwickel, ${ }^{52}$ Lieb, ${ }^{177}$ and Rosenbljum: $: 220$

$$
N(V) \leqslant L_{0, \nu} \int|V(x)|^{\nu / 2} d x \quad(\nu \geqslant 3) .
$$


One reason this is of special interest is that for nice $V^{\prime}$ s as $\lambda \rightarrow \infty, N(\lambda V) / \int|\lambda V|^{v / 2} d x$ converges to a universal constant (see Sec. XIV). In particular, (VIII.1) has the wrong large $\lambda$ behavior while (VIII.2) has the right such behavior. (Simon ${ }^{243}$ had the first bounds with the right large $\lambda$ behavior for nice enough $V$ 's; he also conjectured (VIII.2).)

As in the one-dimensional case, there are Lieb-Thirring-type bounds on the moments of the negative eigenvalues $e_{j}$ of $-\Delta+V$

$$
\sum_{j}\left|e_{j}\right|^{\gamma} \leqslant L_{\gamma, \nu} \int d x|V(x)|^{\gamma+\nu / 2} d^{\nu} x
$$

for $\gamma>0$ if $\nu=2$ and $\gamma \geqslant 0$ if $\nu \geqslant 3$. These were proven first in Lieb and Thirring. ${ }^{185}$ There has been considerable literature on the best values of $L_{\gamma, \nu}$. In particular, a recent pair of papers of Laptev and Weidl ${ }^{168}$ and Hundertmark et al. ${ }^{124}$ has obtained a breakthrough in understanding the $\nu$ dependence of $L_{\gamma, \nu}$. In particular, they show that for $\gamma \geqslant \frac{3}{2}, L_{\gamma, \nu}$ is given by the quasiclassical value. On the other hand, it is known that $L_{\gamma=0, \nu}>L_{\gamma=0, \nu}^{\text {q.c. }}$, the quasiclassical value for all $\nu$ (Helffer and Robert ${ }^{109,110}$ ).

For a review of the literature on bounds on the number of eigenvalues, especially the subtle two-dimensional case, see Birman and Solomyak. ${ }^{36}$

The absence of eigenvalues at positive energies is a specialized issue largely independent of the rest of the analysis of positive spectrum. Given the examples of Wigner-von Neumann and related ones of Naboko and Simon discussed in Sec. V, one needs some condition on the falloff or lack of oscillations. Here is a simple result:

Theorem VIII.2: Let $V(x)=V_{1}(x)+V_{2}(x)$ where $|x|\left|V_{1}(x)\right| \rightarrow 0$ and $\left|(x \cdot \nabla) V_{2}(x)\right| \rightarrow 0$. Then $-\Delta+V$ has no eigenvalues in $[0, \infty)$.

Remarks:

(1) The stated theorem requires local regularity ( $V_{1}$ bounded near infinity and $V_{2}$ is $C^{1}$ ), but there are extensions that allow local singularities.

(2) Rellich ${ }^{216}$ proved that if $V$ has compact support, there are no positive energy eigenvalues. Theorem VIII.2 when $V_{2}=0$ is due to Kato ${ }^{150}$ and the full result to Agmon ${ }^{2}$ and Simon. ${ }^{235}$

(3) See Froese et $\mathrm{l}^{86}{ }^{8}$ for another result of this genre; we will discuss their result further in Sec. IX.

The methods we will discuss below typically show that $\sigma_{\mathrm{pp}} \cap(0, \infty)$ is finite; one can then usually use Theorem VIII.2 to prove that the set is actually empty.

As for positive spectrum, it is intimately related to scattering theory. Given two self-adjoint operators $A, B$, one says the wave operators exist if

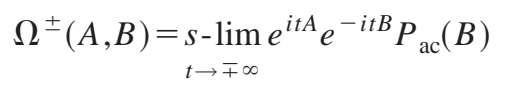

exists where $P_{\mathrm{ac}}$ is the projection onto the a.c. subspace for $B$. We say they are complete if $\operatorname{Ran} \Omega^{ \pm}(A, B)=\operatorname{Ran} P_{\mathrm{ac}}(A)$, in which case $\Omega^{ \pm}(A, B)$ are unitary maps of $\operatorname{Ran} P_{\mathrm{ac}}(B)$ to $\operatorname{Ran} P_{\mathrm{ac}}(A)$ which intertwine $A$ and $B$. See Reed and Simon, ${ }^{213}$ Baumgärtel and Wollenberg, ${ }^{25}$ or Yafaev $^{277}$ (or many other books) for a discussion of the physics involved.

The development of abstract scattering theory is closely intertwined (pun intended) to its applications to Schrödinger operators. Fundamental work was done by Jauch, ${ }^{133}$ Cook, ${ }^{51}$ Rosenblum, ${ }^{221}$ Kato, ${ }^{149}$ Birman, ${ }^{33}$ and Birman and Krein. ${ }^{35}$

The basic result for positive spectrum for "short-range" potentials is:

Theorem VIII.3: Let $V$ be such that $(1+|x|)^{1+\varepsilon} V(x) \in L^{p}+L^{\infty}\left(\mathbb{R}^{\nu}\right)$ for $\max (2, \nu / 2)<p$ $<\infty$ and let $H=-\Delta+V$ and $H_{0}=-\Delta$. Then $\Omega^{ \pm}\left(H, H_{0}\right)$ exist and are complete. Moreover, $H$ has no singular continuous spectrum and any eigenvalues in $(0, \infty)$ are isolated (from other eigenvalues) and of finite multiplicity.

Remarks:

(1) The first results on absence of singular continuous spectrum depended on eigenfunction expansions and were obtained by Povzner ${ }^{209}$ ( $V$ 's of compact support) and Ikebe ${ }^{129}$ ( $V$ 's which 
were $O\left(|x|^{-2-\varepsilon}\right)$ at infinity). The earliest results on completeness of wave operators depended on the trace class theory of scattering (of Rosenblum ${ }^{221}$ and Kato ${ }^{149}$ ) and were obtained by Kuroda. ${ }^{166,167}$ From 1960 to 1972 , the decay was successively improved until Agmon ${ }^{3}$ obtained the $O\left(|x|^{-1-\varepsilon}\right)$ result quoted.

(2) Enss $^{77}$ has a different, quite physical, approach to this result. Enss' work depends in part on an earlier geometric characterization of the continuous subspace of a Schrödinger operator by Ruelle $^{222}$ and Amrein and Georgescu. ${ }^{14}$ This is sometimes called the RAGE theorem after the initials of the authors.

(3) It is known (e.g., Dollard $\left.{ }^{69}\right)$ that if $V(x)=O\left(|x|^{-1}\right), \Omega^{ \pm}\left(H, H_{0}\right)$ may not exist.

For long-range behavior decaying slower than $O\left(|x|^{-1}\right)$, there are results if $\nabla V$ decays faster than $O\left(|x|^{-1-\varepsilon}\right)$. Basically, there is only a.c. spectrum at positive energy if $V=V_{1}+V_{2}$ with $V_{1}=O\left(|x|^{-1-\varepsilon}\right)$ and $x \cdot \nabla V_{2}=O\left(|x|^{-\varepsilon}\right)$. For details, see Lavine, ${ }^{172}$ Agmon and Hörmander, ${ }^{6}$ and Hörmander. ${ }^{122}$ These works use modified wave operators as introduced by Dollard. ${ }^{69}$

\section{N-BODY HAMILTONIANS}

Let $\widetilde{H}$ be the Hamiltonian of $N$ particles in $\mathbb{R}^{\nu}$. Explicitly, $\widetilde{H}$ is an operator on $L^{2}\left(\mathbb{R}^{\nu N}\right)$ given by $\widetilde{H}=\widetilde{H}_{0}+V$ where

$$
\tilde{H}_{0}=-\sum_{j=1}^{N} \frac{1}{2 m_{j}} \Delta_{x_{j}}
$$

with $x=\left(x_{1}, \ldots, x_{N}\right)$ a point in $\mathbb{R}^{\nu N}=\mathbb{R}^{\nu} \times \mathbb{R}^{\nu} \times \ldots \times \mathbb{R}^{\nu}(N$ times $)$ and

$$
V=\sum_{i<j} V_{i j}\left(x_{i}-x_{j}\right)
$$

with $V_{i j}$ a function in $\mathbb{R}^{N}$ which decays at infinity. There is a standard way of removing the center of mass and getting an associated Hamiltonian $H$ on $L^{2}\left(\mathbb{R}^{\nu(N-1)}\right)$. For a more extensive review of the subject than this brief discussion, see Hunziker and Sigal. ${ }^{12}$

For any partition $a$ of $\{1, \ldots, N\}$ into disjoint subsets, one defines $I(a)=\Sigma_{(i, j)} \sqsubset a V_{i j}$ over the pairs $(i, j)$ in distinct clusters and $H(a)=H-I(a)$.

The issues one faces are similar to those in the two-body case but often more subtle. The first thing one needs to establish about $N$-body systems is where the essential spectrum of $H$ lies. The result involves

$$
\begin{gathered}
\Sigma(a)=\inf \operatorname{spec}(H(a)), \\
\Sigma=\min _{\# a \geqslant 2}(\Sigma(a)) .
\end{gathered}
$$

$\Sigma$ is the minimum energy the system can have after it is broken into two pieces moved very far from each other. That makes the following physically attractive:

Theorem IX.1: (HVZ Theorem) Suppose each $V_{i j}$ viewed as an operator on $L^{2}\left(\mathbb{R}^{\nu}\right)$ obeys $V_{i j}\left(-\Delta_{i j}+1\right)^{-1}$ is compact. Then

$$
\sigma_{\text {ess }}(H)=[\Sigma, \infty)
$$

Remarks:

(1) The name "HVZ', comes from work of Hunziker, ${ }^{126}$ van Winter, ${ }^{269}$ and Zhislin ${ }^{280}$ who first proved it.

(2) The original proofs used resolvent equations; a geometric proof was later found by Enss ${ }^{76}$ and Simon. ${ }^{244}$ 
The next issue is whether the discrete spectrum is finite or infinite. A great deal of attention has been paid to atomic or ionic Hamiltonians. Define on $L^{2}\left(\mathbb{R}^{3 N}\right)$ :

$$
H_{M}(N, Z)=\sum_{i=1}^{N}\left(-\Delta_{i}-\frac{Z}{\left|x_{i}\right|}\right)+\frac{1}{M} \sum_{i<j} \nabla_{i} \nabla_{j}+\sum_{i<j} \frac{1}{\left|x_{i}-x_{j}\right|},
$$

which describes $N$ electrons moving around a nucleus of charge $Z$ and mass $M$. A basic result states that neutral atoms and positive ions always have an infinite number of bound states:

Theorem IX.2: $\left(Z_{\text {Zhislin }}^{280}\right)$ If $N \leqslant Z, \operatorname{dim} E_{(-\infty, \Sigma)}\left(H_{M}(N, Z)\right)=\infty$ for any $M$ (including $M$ $=\infty)$.

Remarks:

(1) The first result of this genre was Kato ${ }^{145}$ who proved the result if $N=Z=2$ and $M=\infty$ (Helium). He did not properly handle $M<\infty$ because he did not use the right coordinate systems. As shown by Simon, ${ }^{236}$ Kato's idea, which involved placing $N-1$ electrons in the ground states for the $N-1$ ion and the $N$ th in a hydrogen-like state around the core, can prove Theorem IX.2.

(2) This result holds even if one adds Fermi statistics (see, e.g., $\operatorname{Simon}^{236}$ ).

(3) If $Z$ is not restricted to be an integer, the proper condition is $N<Z+1$.

As for negative ions, we have

Theorem IX.3: $\left(\right.$ Zhislin $\left.^{281}\right) \operatorname{dim} E_{(-\infty, \Sigma)}\left(H_{M}(Z+1, Z)\right)<\infty$

Remarks:

(1) This result also has a geometric proof by Sigal ${ }^{231}$ and Simon. ${ }^{244}$

(2) This result may not be true for fermion electrons because the $N-1$ problem may have a degenerate ground state which allows one with a nonzero dipole moment.

(3) While it is presumably true that $\operatorname{dim} E_{(-\infty, \Sigma)}\left(H_{M}(N, Z)\right)<\infty$ for all $N \geqslant Z+1$, that is not known.

Finally, with regard to bound states of atoms, there is the issue of when $\operatorname{dim} E_{(-\infty, \Sigma)}=0$. The result is the following:

Theorem IX.4: Let $M=\infty$.

(a) (Ruskai ${ }^{223,224}$ and $\operatorname{Sigal}^{231,232}$ ) For any $Z$, there is an $N_{0}(Z)$ so that for $N \geqslant N_{0}(Z)$, there is no spectrum in $(-\infty, \Sigma) . N_{0}(Z)$ denotes the smallest $N_{0}$ for which this is true.

(b) (Lieb et al. ${ }^{181}$ ) For fermions, $N_{0}(Z) / Z \rightarrow 1$ as $Z \rightarrow \infty$.

(c) (Benguria and $\mathrm{Lieb}^{28}$ ) Without Fermi statistics, $N_{0}(Z)>1.2 Z$ for Z large.

(d) $\left(\mathrm{Lieb}^{178}\right) N_{0}(Z) \leqslant 2 Z$.

Remarks:

(1) If $N \geqslant N_{0}$, then inf $\operatorname{spec}(H(N, Z))=\inf \operatorname{spec}\left(H\left(N_{0}, Z\right)\right)<\inf \operatorname{spec}\left(H\left(N_{0}-1, Z\right)\right)$.

(2) Some of these results hold if $M<\infty$.

With short-range potentials, the situation is simple if the bottom of the essential spectrum is two body. Define

$$
\Sigma_{3}=\min _{\#(a) \geqslant 3}(\Sigma(a)) .
$$

Then (see Cycon et al., ${ }^{53}$ Sec. 3.9)

Theorem IX.5: $\left(\right.$ Sigal $\left.^{231}\right)$ Suppose $\Sigma_{3}>\Sigma, \nu \geqslant 3$, and each $V_{i j}$ lies in $L^{\nu / 2}\left(\mathbb{R}^{\nu}\right)$. Then $\operatorname{dim} E_{(-\infty, \Sigma)}(H)<\infty$.

On the other hand, if $\Sigma_{3}=\Sigma$, there can be an infinite number of bound states even if the $V_{i j}$ 's have compact support (in $x_{i j}$ ). In particular, if $N=3, V_{12}=V_{23}=V_{13}=-c \chi_{1}$, with $\chi$ the characteristic function of a unit ball and $c$ chosen so that inf $\operatorname{spec}(H)=0$ but inf $\operatorname{spec}(H+\varepsilon V)<0$ for all $\varepsilon>0$, it is known that $\operatorname{dim} E_{(-\infty, 0)}(H)=\infty$. This is known as the Efimov effect after work of Efimov. ${ }^{74,75}$ For proofs of this phenomenon, see Yafaev ${ }^{276}$ and Ovchinnikov and Sigal. ${ }^{201}$

In analyzing the spectrum of $H$ on $[\Sigma, \infty)$, a particular class of physically significant energies occurs, the thresholds. For each partition $a$ of $\{1, \ldots, N\}$ with $\sharp a \geqslant 2$, there is a natural decompo- 
sition of $L^{2}\left(\mathbb{R}^{\nu(N-1)}\right)=\mathcal{H}_{a} \otimes \mathcal{H}^{a}$ where $\mathcal{H}_{a}$ are functions of $x_{i}-x_{j}$ with $i$ and $j$ in the same cluster of $a$ and $\mathcal{H}^{a}$ are functions of $R_{\alpha}-R_{\beta}$, where $R_{\alpha}$ is the center of mass of a cluster (see Ref. 128 for an elegant way of doing this kinematics). Under the decomposition $H(a)=H_{a} \otimes I+I \otimes T^{a} . H_{a}$ is the internal energy of the cluster and $T^{a}$ the kinetic energy of the cluster centers of mass. $\mathcal{I}(a)$ is the set of eigenvalues of $H_{a}$ (with the condition that if $\#(a)=N$, so $H_{a}$ is 0 on $\mathrm{C}$, then $\mathcal{I}(a)$ $=\{0\}$. The set of thresholds is defined as

$$
\mathcal{I}=\bigcup_{a} \mathcal{I}(a)
$$

Note: An energy in $\mathcal{I}(a)$ is a sum of eigenvalues of individual cluster Hamiltonians. In particular, the statement in the theorems below that the set of thresholds is a closed countable set follows by induction from the other statement that eigenvalues can only accumulate at thresholds.

The three-body problem turns out to have some aspects that make it simpler than the general $N$-body problem, and Faddeev ${ }^{79}$ and later Enss ${ }^{78}$ (using very different methods) have fairly complete results on spectral and scattering theory for $N=3$. We will focus here on results that apply for all $N$.

Historically, the first aspect of the continuous spectrum for general $\mathrm{N}$-body systems controlled was the absence of a singular continuous spectrum. The earliest result required analyticity of the potentials but included atoms:

Theorem IX.6: (Balslev and Combes $\left.{ }^{23}\right)$ Suppose each $V_{i j}(x)=f_{i j}\left(x_{i}-x_{j}\right)$ where $f_{i j}$ is a function on $\mathbb{R} \backslash\{0\}$ that obeys

$$
A(\theta)=V\left(e^{\theta} x\right)(-\Delta+1)^{-1}
$$

is compact and has an analytic continuation from $\theta \in \mathbb{R}$ to $\{\theta|| \operatorname{Im} \theta \mid<\varepsilon\}$ for some $\varepsilon>0$. Then $\sigma_{\mathrm{sc}}(H)=\varnothing$.

Moreover,

(i) Any eigenvalue of $H$ in $R \backslash \mathcal{I}$ is of finite multiplicity, and eigenvalues can only accumulate at thresholds.

(ii) The set of eigenvalues union thresholds is a closed countable set.

\section{Remarks:}

(1) Such potentials are called dilation analytic.

(2) This result was first proven for two-body systems by Aguilar and Combes. ${ }^{7}$

(3) See $\operatorname{Simon}^{237,238}$ for extensions of this result.

The most general results on absence of singular continuous spectrum depend on the ideas of Mourre. ${ }^{196}$

Theorem IX.7: Suppose $V_{i j}(x)=f_{i j}\left(x_{i}-x_{j}\right)$ where $f_{i j}$ is a function on $\mathbb{R}^{\nu}$ that obeys (as operators on $\left.L^{2}\left(\mathbb{R}^{\nu}\right)\right)$

(i) $\quad f_{i j}(x)(-\Delta+1)^{-1}$ is compact;

(ii) $\quad(-\Delta+1)^{-1} x \cdot \nabla f_{i j}(-\Delta+1)^{-1}$ is compact.

Then $\sigma_{\text {ess }}(H)$ is empty. Moreover, any eigenvalue in $\mathrm{R} \backslash \mathcal{I}$ is discrete, eigenvalues can only accumulate at thresholds, and the set of eigenvalues and thresholds is a closed countable set.

Remarks:

(1) This theorem was proven for $N=3$ by Mourre. ${ }^{196}$ His methods were extended and elucidated by Perry et al. ${ }^{205}$ who obtained the general $N$-body result. Substantial simplifications of the proof were found by Froese and Herbst. ${ }^{85}$

(2) Condition (ii) does not require that $f_{i j}$ be smooth because $\nabla f_{i j}=\left[\nabla, f_{i j}\right]$ and $\nabla(-\Delta$ $+1)^{-1}$ is bounded. Basically, (i), (ii) hold if $f_{i j}=f_{i j}^{(1)}+f_{i j}^{(2)}$, where $x f_{i j}^{(1)}(-\Delta+1)^{-1}$ is compact and $f_{i j}^{(2)}$ is smooth with $(x \cdot \nabla) f_{i j}^{(2)}(-\Delta+1)^{-1}$ and $f_{i j}^{(2)}(-\Delta+1)^{-1}$ compact. 
(3) Froese and Herbst ${ }^{85}$ have some general results that imply that $\mathcal{I} \cap(0, \infty)=\varnothing$ (see Theorem 4.19 in Cycon et al. ${ }^{53}$ ).

Finally, there has been extensive study of scattering theory and completeness. For each cluster with $\sharp(a) \geqslant 2$, let $P_{a}$ on $\mathcal{H}_{a}$ be the projection onto the point spectrum of $H_{a}$ and let $P(a)=P_{a}$ $\otimes I$, the projection onto vectors which are bound within the clusters and arbitrary for the centers of mass coordinates. The cluster wave operators are defined by

$$
\Omega^{ \pm}(a)=\underset{\substack{s-\lim _{t \rightarrow \infty} \\ e^{+i t H}}}{-i t H(a)} P(a) .
$$

$\operatorname{Ran}\left(\Omega^{+}(a)\right)$ are those states which in the distant past look like bound clusters (corresponding to the partition $a$ ) moving freely relative to one another.

The existence of cluster wave operators (IX.3) was proven first by Hack. ${ }^{103}$ It is not hard to see (e.g., Theorem XI.36 in Reed and Simon ${ }^{213}$ ) that for $a \neq b$, Ran $\Omega^{+}(a)$ is orthogonal to Ran $\Omega^{+}(b)$. Asymptotic completeness is the statement that

$$
\underset{\#(a) \geqslant 2}{\oplus} \operatorname{Ran}\left(\Omega^{+}(a)\right)=\mathcal{H}_{\mathrm{ac}}(H),
$$

where $\mathcal{H}_{\mathrm{ac}}(H)$ is the absolutely continuous subspace for $H$. After fairly general results for $N=3$ $\left(\right.$ Faddeev $^{79}$ and Enss ${ }^{78}$ ) and for general $N$ with weak coupling (Iorio and O'Carroll ${ }^{131}$ ) and repulsive potentials (Lavine ${ }^{171}$ ), Sigal and Soffer ${ }^{233}$ solved the general result. Their theorem is

Theorem IX.8: (Sigal and Soffer $\left.{ }^{233}\right)$ If each $V_{i j}(x)=f_{i j}\left(x_{i}-x_{j}\right)$ where $\mid\left(D^{\alpha} f_{i j}(x) \mid \leqslant C(1\right.$ $+|x|)^{-|\alpha|-\varepsilon^{-1}}$ for all multiindices with $|\alpha| \leqslant 2$, then asymptotic completeness holds.

Extensions and clarifications of this work are due to Graf, ${ }^{101}$ Hunziker, ${ }^{127}$ and Yafaev. ${ }^{278}$ Long-range potentials are discussed in Derezinski, ${ }^{65}$ Sigal and Soffer, ${ }^{234}$ and Derezinski and Gerard. ${ }^{66}$

\section{CONSTANT ELECTRIC AND MAGNETIC FIELDS}

Quantum mechanics with a potential and constant electric or magnetic field played a critical role experimentally and theoretically in the earliest days of the subject, and there has been considerable mathematical literature on the spectral properties of these operators. The basic Stark Hamiltonian on $L^{2}\left(\mathbb{R}^{\nu}\right)$ is

$$
H=-\Delta+E x_{1}+V(x),
$$

where $V$ is short range. A key role has been played by an explicit formula of Avron and Herbst ${ }^{18}$ for the operator when $V=0$, viz.,

$$
\exp \left(-i t\left(-\Delta+x_{1}\right)\right)=\exp \left(-i t^{3} / 3\right) \exp \left(-i t x_{1}\right) \exp \left(-i t \Delta+i p_{1} t^{2}\right),
$$

where $p_{1}=(1 / i)\left(\partial / \partial x_{1}\right)$. Classically in an electric field, a particle has $x_{1}=N-c t^{2}$ as $t \rightarrow \infty$ and (X.2) realizes this with the $p_{1} t^{2}$ term. It means the borderline for short range is $|x|^{-1 / 2-\varepsilon}$ rather than $|x|^{-1-\varepsilon}$. The result is

Theorem X.1: Suppose $|V(x)| \leqslant C(1+|x|)^{-\varepsilon}\left(1+\left|x_{1}\right|\right)^{-1 / 2-\varepsilon}$. Then $H$ given by (X.1) has complete wave operators and empty singular continuous spectrum. Eigenvalues are isolated and of finite multiplicity.

This result and ones similar to it are discussed by Herbst, ${ }^{114}$ Yajima, ${ }^{279}$ and Simon. ${ }^{249}$ Multiparticle completeness in electric fields has been studied by Herbst et al.. ${ }^{116}$ and Adachi and Tamura. ${ }^{1}$

There is much literature on both constant and variable magnetic fields but an extensive review of it is beyond the scope of this paper. One can begin looking at the literature by consulting a series by Avron et al. ${ }^{19-21}$ and Chapter 6 of Cycon et al. ${ }^{53}$ and references therein. See also Sec. XII. 


\section{COULOMB ENERGIES}

While much of the mathematical theory of nonrelativistic quantum mechanics has focused on general potentials, nature uses the Coulomb potential and there is considerable literature on binding energies of Coulomb systems, especially as some parameter goes to infinity. Section IX (see Theorem IX.4) already discussed one such result. We will only introduce some seminal themes; consult Lieb ${ }^{179}$ for a review of the subject.

The most famous of these results is the stability of matter. In its simplest form, it concerns the Hamiltonian

$$
H\left(N, M ; R_{1}, \ldots, R_{M}\right)=-\sum_{i=1}^{N} \Delta_{i}-\sum_{i, \alpha} \frac{1}{\left|x_{i}-R_{\alpha}\right|}+\sum_{i<j} \frac{1}{\left|x_{i}-x_{j}\right|}+\sum_{\alpha<\beta} \frac{1}{\left|R_{\alpha}-R_{\beta}\right|}
$$

of $N$ electrons moving in the field of $M$ infinitely massive protons. Let $\mathcal{H}_{f}$ be the functions on $L^{2}\left(\mathbb{R}^{3 N}\right)$ thought of as functions $\psi\left(x_{1}, \ldots, x_{N}\right)$ of $N$ variables in $\mathbb{R}^{3}$ which are antisymmetric, that is,

$$
\psi\left(x_{\pi(1)}, \ldots, x_{\pi(n)}\right)=(-1)^{\pi} \psi\left(x_{1}, \ldots, x_{N}\right)
$$

for any permutation $\pi$; that is, $\mathcal{H}_{f}$ is the wave function with Fermi statistics (we ignore spin which is easily accommodated). Define

$$
E(N, M)=\inf _{\substack{\psi \in \mathcal{H}_{f} \\ R_{1}, \ldots, R_{M}}}\left\langle\psi, H\left(N, M ; R_{1}, \ldots, R_{M}\right) \psi\right\rangle
$$

Stability of matter states that

$$
E(N, M) \geqslant-c(N+M) .
$$

Among other things, this bound is important because it is equivalent to the fact that the radius of a chunk of matter with $N=M$ does not shrink to zero as $N \rightarrow \infty$.

The first proof of (XI.1) was obtained by Dyson and Lenard ${ }^{72,73}$ with a constant $C$ that was many powers of ten too large. Lieb and Thirring ${ }^{186}$ found an elegant proof with a constant $C$ that is on the order of magnitude of Rydbergs. The result (XI.1) fails if one does not impose Fermi statistics (see Dyson ${ }^{71}$ and Conlon et al. ${ }^{50}$ ). Extensions that involve relativistic kinetic energy, magnetic and/or radiation fields can be found in Conlon, ${ }^{49}$ Lieb et al. ${ }^{180}$ and Fefferman et al. ${ }^{81}$

Another Coulomb energy problem that has been extensively studied is the total binding energy in the limit of large of $Z$. One defines

$$
H(N, Z)=\sum_{i=1}^{N}\left(-\Delta_{i}-\frac{Z}{\left|x_{i}\right|}\right)+\sum_{i<j} \frac{1}{\left|x_{i}-x_{j}\right|}
$$

on $\mathcal{H}_{f}$ and

$$
E(N, Z)=\inf _{\psi \in \mathcal{H}_{f}}\langle\psi, H(N, Z) \psi\rangle
$$

and

$$
E(Z)=\min _{N} E(N, Z) .
$$

One knows that

$$
E(Z)=\alpha Z^{7 / 3}+\beta Z^{2}+\gamma Z^{5 / 3}+o\left(Z^{5 / 3}\right) .
$$


The $\alpha$ term is given by Thomas-Fermi theory and this leading asymptotics was proven by Lieb and Simon. ${ }^{182}$ The $\beta$ term is called the Scott correction and it was established by a combination of ideas of Hughes ${ }^{123}$ and Siedentop and Weikard. ${ }^{229,230}$ The full asymptotics (XI.2) was obtained by Fefferman and Seco. ${ }^{82}$ Results for large $Z$ and large magnetic field can be found in Lieb et al. ${ }^{183,184}$

\section{EIGENVALUE PERTURBATION THEORY}

Some of Schrödinger's earliest papers on quantum mechanics concerned eigenvalue perturbation theory. Kato's book ${ }^{153}$ is a source of detailed information on what we will call regular and asymptotic perturbation theory below. A review of some of the other aspects can be found in Reed and Simon ${ }^{212}$ and Simon. ${ }^{254}$

If $A$ is self-adjoint and $B$ is $A$-bounded in the sense of (III.3), and if $E_{0}$ is a simple eigenvalue of $A$, then for $\beta$ small, $A+\beta B$ has a unique eigenvalue $E(\beta)$ near $E_{0}$ and $E(\beta)$ is analytic in $\beta$. This is a result of Rellich ${ }^{215}$ and Kato. ${ }^{142,143}$ An example is

$$
-\Delta_{1}-\Delta_{2}-\frac{1}{\left|x_{1}\right|}-\frac{1}{\left|x_{2}\right|}-\frac{1}{Z} \frac{1}{\left|x_{1}-x_{2}\right|}
$$

about $1 / Z=0$ which is equivalent after scaling (of space and energy) to

$$
-\Delta_{1}-\Delta_{2}-\frac{Z}{\left|x_{1}\right|}-\frac{Z}{\left|x_{2}\right|}+\frac{1}{\left|x_{1}-x_{2}\right|} \text {. }
$$

The numerical radius of convergence in $|1 / Z|$ is about 1.06 so $H(Z=2)$ and $H(Z=1)$ are both included. Kato ${ }^{147}$ developed the theory for form perturbations. Rellich and Kato included degenerate eigenvalues.

Titchmarsh $^{265,266}$ and Kato ${ }^{146}$ also developed the theory of asymptotic situations like the anharmonic oscillator

$$
-\frac{d^{2}}{d x^{2}}+x^{2}+\beta x^{4}
$$

where each eigenvalue $E_{n}(\beta)$ for $\beta>0$ has an asymptotic series

$$
E_{n}(\beta) \sim \sum_{n=0}^{\infty} a_{n} \beta^{n}
$$

even though this series can be divergent (and is for the case (XII.2), as shown by Bender and $\mathrm{Wu}^{27}$ ). See Herbst and Simon ${ }^{117}$ for an example where an asymptotic series converges but to the wrong answer! See Simon ${ }^{252}$ for a study of multiwell problems.

In some cases, including (XII.2), it is known that the divergent perturbation series can be made to give the right eigenvalue with a summability method, either Padé approximation (Loeffel et al. ${ }^{187}$ ) or Borel summation (Graffi et al. ${ }^{102}$ ). Borel summability is also known to work for the Zeeman series for hydrogen-hydrogen perturbed by turning on a constant magnetic field; see Avron et $a l^{21}$ and Avron et al. ${ }^{17}$

In certain cases, eigenvalues are perturbed into resonances, the subject of Sec. XIII. For eigenvalues embedded in continuous spectrum under regular perturbations (like (XII.1)), the convergence of the perturbation series for a resonance and its related time-dependent perturbation theory and the Fermi golden rule is discussed in Simon. ${ }^{237,238}$ For Stark Hamiltonians, the basic paper is Herbst. ${ }^{115}$ Harrell and Simon ${ }^{107}$ found the leading resonance asymptotics in this case. 


\section{RESONANCES}

Almost everything we have discussed so far has involved a single operator and properties invariant under unitary transformations. The notion of resonances has got to involve additional structure. For example, the operators $-\Delta-|x|^{-1}-F x=H(F)$ are unitarily equivalent for all $F$ $\neq 0$. But according to the physics lore, there is a resonance with an $F$-dependent position. We will not emphasize the extra structure, but it is there. We will focus on two definitions of resonances: one suitable for potentials that decay very rapidly (see Zworski ${ }^{284,285}$ for reviews) and the method of complex scaling already discussed in a different context in Sec. IX. (See Reed and Simon ${ }^{212}$ and Simon $^{245}$ for reviews.)

Let $\nu$ be an odd dimension, let $V$ be a bounded potential of compact support on $\mathbb{R}^{\nu}$, and for Re $\kappa>0$, define

$$
B(\kappa)=|V|^{1 / 2}\left(-\Delta+\kappa^{2}\right)^{-1} V^{1 / 2},
$$

where $V^{1 / 2}=|V|^{1 / 2} \operatorname{sgn}(V)$. Then $-\kappa^{2}$ is an eigenvalue of $-\Delta+V$ if and only if -1 is an eigenvalue of $B(\kappa)$. Since $\nu$ is odd, $B(\kappa)$ has an analytic continuation as a compact operator-valued function of $\kappa$ to all of $\mathrm{C}$ (when $\nu=1$, there is a simple pole at $\kappa=0$ but $\kappa B(\kappa)$ is entire). If $\operatorname{Re} \kappa<0$ and -1 is an eigenvalue of $B(\kappa)$, we say $-\kappa^{2}$ is a resonance of $-\Delta+V$.

Froese $^{83}$ has a lovely formula that relates resonances defined by this method to scattering theory. For all $\kappa, B(\kappa)-B(-\kappa)$ is trace class so $(1+B(-\kappa))(1+B(\kappa))^{-1}$ is 1 plus trace class and has a determinant as an operator on $L^{2}\left(\mathbb{R}^{\nu}\right)$. For $k$ real and $S(k)$, the $S$ matrix on $L^{2}\left(S^{\nu-1}\right)$,

$$
\operatorname{det}(S(k))=\operatorname{det}\left((1+B(-i k))(1+B(i k))^{-1}\right),
$$

so resonances are related to poles of the analytic continuation of $S$.

There has been considerable literature on the number of resonances. Let $N(R)$ be the number of resonances with energy $E$ obeying $|E|<R$. In one dimension, one has a complete result:

Theorem XIII.1: (Zworski ${ }^{282}$ ) Let $\nu=1$ and suppose $[a, b]$ is the convex hull of the support of $V$. Then

$$
\lim _{R \rightarrow \infty} R^{-1 / 2} N(R)=\frac{2}{\pi}|b-a|
$$

Remarks:

(1) The result depends on a theorem of Titchmarsh and Cartwright on the zeros of Fourier transforms of functions of compact support.

(2) Froese $^{83}$ has obtained some results for cases when a potential decays faster than any exponential but may not have compact support.

In higher dimensions, much less is known. Zworski ${ }^{283}$ proved that for $V$ of compact support, $N(R) \leqslant C(R+1)^{\nu / 2}$ (see also Froese ${ }^{84}$ ). On the other hand for general $V$ 's, it is only known (Sá Barreto and Zworski ${ }^{225}$ ) that $\lim _{R \rightarrow \infty} N(R)=\infty$.

Suppose $V$ is a dilation analytic potential in the sense of Theorem IX.6. Let

$$
H(\theta)=-e^{-2 \theta} \Delta+V\left(e^{\theta} \tau\right) .
$$

Because of the analyticity assumption, $H(\theta)$ is analytic in $\{\theta|| \operatorname{Im}(\theta) \mid<\alpha\}$ for some $\alpha$. Then Aguilar and Combes ${ }^{7}$ found the essential spectrum of $H(\theta)$ for $N=2$ and Balslev and Combes ${ }^{23}$ for general $N$ :

Theorem XIII.2: $\sigma_{\text {ess }}(H(\theta))=\cup_{E \in \mathcal{I}(\theta)}\left(E+e^{-2 \theta} \mathbb{R}\right)$

Remarks:

(1) $\mathcal{I}(\theta)$ is the thresholds of $H(\theta)$ defined analogously to the case $\theta=0$. It is not hard to see that $\sigma_{\text {ess }}(H(\theta))$ and $\mathcal{I}(\theta)$ depend only on $\operatorname{Im} \theta$.

(2) If $\operatorname{Im} \theta>0, \sigma_{\text {ess }}(H(\theta)) \cap \mathbb{R}$ consists precisely of $\mathcal{I}$. Basically as we increase $\operatorname{Im} \theta$ from 0 , the essential spectrum rotates about the thresholds. In doing that, it can uncover resonances. 
Resonances defined by this method have been used by quantum chemists for numerical calculations as well as a theoretical tool. Simon ${ }^{237,238}$ used it to study the Fermi golden rule and Harrell and Simon ${ }^{107}$ to prove various one-dimensional tunneling estimates.

Avron ${ }^{16}$ used these ideas to study large-order perturbation theory for hydrogen in a magnetic field; a rigorous proof of his results was obtained by Helffer and Sjöstrand. ${ }^{112}$

Herbst ${ }^{115}$ has extended the ideas to Hamiltonians with constant electric field. Among his results is the surprising one that if $0<\operatorname{Im} \theta<\pi / 3$, then $-e^{-2 \theta} \Delta+e^{\theta} x$ has empty spectrum!

\section{THE QUASICLASSICAL LIMIT}

There has been considerable literature on the connection between quantum and classical mechanics. Much of it has focused on what happens as $\hbar \rightarrow 0$, but there are other limiting situations where a classical or semiclassical picture is appropriate-for example, the large $Z$ limit of atoms. We will touch on some of the subjects considered, but the literature is vast. Robert ${ }^{219}$ has an excellent review of those results obtained for very smooth potentials using the Fourier integral operator methods pioneered by Hörmander and Maslov. Therefore, I will not try to cover these results here. We note that in Sec. XI, we referenced the Thomas-Fermi limit, which is quasiclassical.

Consider first the $\hbar \downarrow 0$ limit. Let $H_{\hbar}=-\left(\hbar^{2} / 2 m\right) \Delta+V$. Kac ${ }^{140,141}$ had the idea that the small $\hbar$ limit of $\exp \left(-s H_{\hbar}\right)$ was the same as the zero time limit in Brownian motion. This allows one to prove under great generality that the quantum partition function $\operatorname{Tr}\left(\exp \left(-s H_{\hbar}\right)\right)$ approaches a classical partition function as $\hbar \downarrow 0$; see, for example, Theorem 10.1 in Simon. ${ }^{246}$ The earliest results I know of on this subject are due to Berezin. ${ }^{31}$

Quantum dynamics, $e^{-i s H_{\hbar} / \hbar} \psi_{\hbar}$, on suitable states $\psi_{\hbar}$ make an elegant classical limit-one takes $\psi_{\hbar}$ to be a coherent state which collapses to a single point in phase space as $\hbar \downarrow 0$. Such results were found by Hagedorn ${ }^{104-106}$ (similar methods were developed independently by Ralston $\left.{ }^{210}\right)$.

Since $-\hbar^{2} \Delta+V=\hbar^{2}\left[-\Delta+\hbar^{-2} V\right]$, the small $\hbar$ limit is the same as the large coupling constant limit for $-\Delta+\lambda V$. In particular, if $N(V)=\operatorname{dim} E_{(-\infty, 0)}(-\Delta+V)$, the quantity discussed in Sec. VIII, one has

Theorem XIV.1: Let $\nu \geqslant 3$ and $V \in L^{\nu / 2}\left(\mathbb{R}^{\nu}\right)$. Then $\lim _{\lambda \rightarrow \infty} N(\lambda V) / \lambda^{\nu / 2}=(2 \pi)^{-\nu} \tau_{\nu} \int_{V \leqslant 0}$ $\left(-V(x)^{\nu / 2} d^{\nu} x\right)$, where $\tau_{\nu}$ is the volume of a unit ball in $\mathbb{R}^{\nu}$.

Remarks:

(1) This theorem is quasiclassical since the right side is $(2 \pi)^{-\nu}$ times the volume of the classical phase space region where $p^{2}+V(x) \leqslant 0$.

(2) The historical thread for this theorem goes back to a celebrated paper of Weyl ${ }^{274}$ on Dirichlet Laplacians. Theorems like XIV.1 with stronger conditions on $V$ are due to Birman and Borzov, ${ }^{34}$ Kac, ${ }^{141}$ Martin,${ }^{191}$ and Tamura. ${ }^{263}$ See Reed and Simon, ${ }^{212}$ Theorem XIII.80) for the proof under the stated assumptions.

Let $V(x) \rightarrow \infty$ as $|x| \rightarrow \infty$ in a fairly regular way (e.g., suppose $V$ is an elliptic polynomial). Then $-\Delta+V$ has discrete spectrum and the asymptotics of the number of eigenvalues $\operatorname{dim} E_{(-\infty, \alpha]}(-\Delta+V)$ as $\alpha \rightarrow \infty$ is determined by phase space. Results of this type go back to Titchmarsh; ${ }^{267}$ see also Reed and Simon, ${ }^{212}$ Theorem XIII.81). Similarly, if $V(x) \rightarrow 0$ but so slowly that $N(V)=\infty$, for example, $V(x) \sim-|x|^{-\beta}$ with $0<\beta<2$, then the divergence of $\operatorname{dim} E_{(-\infty, \alpha]}(-\Delta+V)$ as $\alpha \uparrow 0$ is sometimes given by quasiclassical considerations; see Brownell and Clark, ${ }^{40}$ McLeod, ${ }^{194}$ and Reed and Simon, ${ }^{212}$ Theorem XIII.82).

\section{ACKNOWLEDGMENTS}

I would like to thank Michael Aizenman, Brian Davies, Percy Deift, Fritz Gesztesy, Dirk Hundertmark, Walter Hunziker, and Rowan Killip for useful input.

This material is based upon work supported by the National Science Foundation under Grant No. DMS-9707661. The Government has certain rights in this material. 


\section{REFERENCES}

This order of references, which departs from the usual practice of this journal, was permitted on an exceptional basis by the editor for the convenience of the reader.

${ }^{1}$ T. Adachi and H. Tamura, "Asymptotic completeness for long-range many-particle systems with Stark effect, II," Commun. Math. Phys. 174, 537-559 (1996).

${ }^{2}$ S. Agmon, "Lower bounds for solutions of Schrödinger-type equations in unbounded domains," Proceedings International Conference on Functional Analysis and Related Topics, University of Tokyo Press, Tokyo, 1969.

${ }^{3}$ S. Agmon, "Spectral properties of Schrödinger operators and scattering theory," Ann. Norm. Sup. Pisa C1 Sci II 2 , $151-218(1975)$

${ }^{4}$ S. Agmon, “Lectures on Exponential Decay of Solutions of Second-Order Elliptic Equations," Princeton University Press, Princeton (1982).

${ }^{5} \mathrm{~S}$. Agmon, "On positive solutions of elliptic equations with periodic coefficients in $R^{N}$, spectral results and extensions to elliptic operators on Riemannian manifolds," in Differential Equations, edited by I. Knowles and R. Lewis (North Holland, Amsterdam, 1984)

${ }^{6} \mathrm{~S}$. Agmon and L. Hörmander, "Asymptotic properties of solutions of differential equations with simple characteristics," J. Anal. Math. 30, 1-38 (1976).

${ }^{7}$ J. Aguilar and J. M. Combes, “A class of analytic perturbations for one-body Schrödinger Hamiltonians," Commun. Math. Phys. 22, 269-279 (1971).

${ }^{8}$ M. Aizenman and G. M. Graf, "Localization bounds for an electron gas," J. Phys. A 31, 6783-6806 (1998).

${ }^{9}$ M. Aizenman and E. Lieb, “On semiclassical bounds for eigenvalues of Schrödinger operators," Phys. Lett. 66A, 427-429 (1978).

${ }^{10}$ M. Aizenman and S. Molchanov, "Localization at large disorder and at extreme energies: An elementary derivation," Commun. Math. Phys. 157, 245-277 (1993).

${ }^{11}$ M. Aizenman, J. H. Schenker, R. M. Friedrich, and D. Hundertmark "Constructive fractional-moment criteria for localization in random operators,"' Physica A (to be published).

${ }^{12}$ M. Aizenman and B. Simon, "Brownian motion and Harnack's inequality for Schrödinger operators," Commun. Pure Appl. Math. 35, 209-271 (1982).

${ }^{13}$ W. Allegretto, "Positive solutions and spectral properties of second order elliptic operators," Pac. J. Math. 92, 15-25 (1981).

${ }^{14} \mathrm{~W}$. Amrein and V. Georgescu, "On the characterization of bound states and scattering states in quantum mechanics," Helv. Phys. Acta 46, 635-658 (1973).

${ }^{15}$ F. V. Atkinson, “'On the location of the Weyl circles," Proc. R. Soc. Edinburgh, Sect. A: Math. 88A, 345-356 (1981).

${ }^{16}$ J. Avron, "Bender-Wu formulas for the Zeeman effect in hydrogen," Ann. Phys. (N.Y.) 131, 73-94 (1981).

${ }^{17}$ J. Avron et al., "The Bender-Wu formula, $\mathrm{SO}(4,2)$ dynamical group and the Zeeman effect in hydrogen," Phys. Rev. Lett. 43, 691-693 (1979).

${ }^{18}$ J. Avron and I. Herbst, "Spectral and scattering theory of Schrödinger operators related to the Stark effect," Commun. Math. Phys. 52, 239-254 (1977).

${ }^{19}$ J. Avron, I. Herbst, and B. Simon, "Schrödinger operators with magnetic fields, I. General interactions," Duke Math. J. 45, 847-883 (1978).

${ }^{20}$ J. Avron, I. Herbst, and B. Simon, "Schrödinger operators with magnetic fields, II. Separation of center of mass in homogeneous magnetic fields," Ann. Phys. (N.Y.) 114, 431-451 (1978).

${ }^{21}$ J. Avron, I. Herbst, and B. Simon, "Schrödinger operators with magnetic fields, III. Atoms in homogeneous magnetic field,' Commun. Math. Phys. 79, 529-572 (1981).

${ }^{22}$ J. Avron and B. Simon, “Almost periodic Schrödinger operators, II. The integrated density of states,” Duke Math. J. 50, 369-391 (1983).

${ }^{23}$ E. Balslev and J. M. Combes, "Spectral properties of many-body Schrödinger operators with dilation-analytic interactions," Commun. Math. Phys. 22, 280-294 (1971).

${ }^{24}$ V. Bargmann, "On the number of bound states in a central field of force," Proc. Natl. Acad. Sci. USA 38, 961-966 (1952).

${ }^{25} \mathrm{H}$. Baumgärtel and M. Wollenberg, Mathematical Scattering Theory (Birkhäuser, Basel, 1983).

${ }^{26}$ E. D. Belokolos, A. I. Bobenko, V. Z. Enol'skii, A. R. Its, and V. B. Matveev, Algebro-Geometric Approach to Nonlinear Integrable Equations (Springer, Berlin, 1994).

${ }^{27}$ C. Bender and T. T. Wu, "Anharmonic oscillator," Phys. Rev. 184, 1231-1260 (1969).

${ }^{28}$ R. Benguria and E. Lieb, "A proof of the stability of highly negative ions in the absence of the Pauli principle,' Phys. Rev. Lett. 50, 1771-1774 (1983).

${ }^{29}$ J. Berezanskii, "'On expansion according to eigenfunctions of general self-adjoint differential operators," Dokl. Akad. Nauk SSSR 108, 379-382 (1956).

${ }^{30}$ J. Berezanskii, "Expansions in eigenfunctions of self-adjoint operators," Transl. Math. Mono. 17, Am. Math. Soc., Providence, RI (1968)

${ }^{31}$ F. A. Berezin, Wick and anti-Wick operator symbols, Mat. Sb. 86, 578-610 (1971) [Math. USSR Sb. 15, 577-606 (1971)].

${ }^{32}$ M. S. Birman, "On the number of eigenvalues in a quantum scattering problem," Vest. LSU 16, 163-166 (1961).

${ }^{33}$ M. S. Birman, "Conditions for the existence of wave operators," Dokl. Akad. Nauk SSSR 143, 506-509 (1962).

${ }^{34}$ M. S. Birman and V. V. Borzov, "On the asymptotics of the discrete spectrum of some singular differential operators," Topics Math. Phys. 5, 19-30 (1972). 
${ }^{35}$ M. S. Birman and M. G. Krein, "'On the theory of wave operators and scattering operators,' Dokl. Akad. Nauk SSSR 144, 475-478 (1962).

${ }^{36}$ M. S. Birman and M. Z. Solomyak, "Estimates for the number of negative eigenvalues of the Schrödinger operator and its generalizations," Advances Soviet Mathematics, edited by M. S. Birman (American Mathematical Society, Providence, RI, 1991), Vol. 7, pp. 1-55.

${ }^{37}$ G. Borg, "Uniqueness theorems in the spectral theory of $y^{\prime \prime}+(\lambda-q(x)) y=0$," Proceedings 11 th Scandinavian Congress of Mathematicians, Johan Grundt Tanums Forlag, Oslo, 1952, pp. 276-287.

${ }^{38}$ J. Bourgain and M. Goldstein, “On nonperturbative localization with quasiperiodic potential," preprint.

${ }^{39}$ F. Browder, "Eigenfunction expansions for formally self-adjoint partial differential operators, I, II," Proc. Natl. Acad. Sci. USA 42, 769-771 (1956); 42, 870-872 (1956).

${ }^{40}$ F. H. Brownell and C. W. Clark, "Asymptotic distribution of the eigenvalues of the lower part of the Schrödinger operator spectrum," J. Math. Mech. 10, 31-70 (1961).

${ }^{41}$ R. Carmona, "Regularity properties of Schrödinger and Dirichlet semigroups," J. Funct. Anal. 17, $227-237$ (1974).

${ }^{42}$ R. Carmona, "One-dimensional Schrödinger operators with random or deterministic potentials: New spectral types," J. Funct. Anal. 51, 229-258 (1983).

${ }^{43}$ R. Carmona, A. Klein, and F. Martinelli, “Anderson localization for Bernoulli and other singular potentials,” Commun. Math. Phys. 108, 41-66 (1987).

${ }^{44}$ R. Carmona and J. Lacroix, Spectral Theory of Random Schrödinger Operators (Birkhäuser, Boston, 1990).

${ }^{45}$ K. Chadan and P. C. Sabatier, Inverse Problems in Quantum Scattering Theory, 2nd ed., (Springer, New York, 1989).

${ }^{46}$ M. Christ and A. Kiselev, "Absolutely continuous spectrum for one-dimensional Schrödinger operators with slowly decaying potentials: Some optimal results," J. Am. Math. Soc. 11, 771-797 (1998).

${ }^{47}$ J. M. Combes and L. Thomas, "Asymptotic behavior of eigenfunctions for multi-particle Schrödinger operators," Commun. Math. Phys. 34, 251-270 (1973).

${ }^{48}$ M. Combescure and J. Ginibre, "Spectral and scattering theory for the Schrödinger operator with strongly oscillating potentials," Ann. Inst. Henri Poincaré, Sect. A A24, 17-29 (1976).

${ }^{49}$ J. Conlon, “The ground state energy of a classical gas," Commun. Math. Phys. 94, 439-458 (1984).

${ }^{50}$ J. Conlon, E. Lieb, and H.-T. Yau, "The $N^{7 / 5}$ law for charged bosons," Commun. Math. Phys. 116, 417-448 (1988).

${ }^{51}$ J. Cook, "Convergence of the Moller wave matrix," J. Math. and Phys. 36, $82-87$ (1957).

${ }^{52}$ M. Cwickel, "Weak type estimates for singular values and the number of bound states of Schrödinger operators," Ann. Math. 106, 93-102 (1977).

${ }^{53}$ H. Cycon, R. Froese, W. Kirsch, and B. Simon, Schrödinger Operators With Application to Quantum Mechanics and Global Geometry (Springer, Berlin, 1987).

${ }^{54}$ E. B. Davies, 'Properties of Green's functions of some Schrödinger operators," J. London Math. Soc. 7, 473-491 (1973).

${ }^{55}$ E. B. Davies, Heat Kernels and Spectral Theory (Cambridge University Press, Cambridge, 1989).

${ }^{56}$ E. B. Davies, " $L^{p}$ spectral theory of higher-order elliptic differential operators," Bull. London Math. Soc. 29, 513-546 (1997).

${ }^{57}$ E. B. Davies and Y. Safarov (eds.), Spectral Theory and Geometry, London Math. Soc. Lecture Notes, Vol. 273 (Cambridge University Press, Cambridge, 1999).

${ }^{58}$ P. Deift and R. Hempel, "On the existence of eigenvalues of the Schrödinger operator $H-\lambda W$ in a gap of $\sigma(H)$," Commun. Math. Phys. 103, 461-490 (1986).

${ }^{59}$ P. Deift, W. Hunziker, B. Simon, and E. Vock, "Pointwise bounds on eigenfunctions and wave packets in $N$-body quantum systems, IV,' Commun. Math. Phys. 64, 1-34 (1978).

${ }^{60} \mathrm{P}$. Deift and R. Killip, " On the absolutely continuous spectrum of one-dimensional Schrödinger operators with square summable potentials,' Commun. Math. Phys. 203, 341-347 (1999).

${ }^{61}$ P. Deift and E. Trubowitz, "Inverse scattering on the line," Commun. Pure Appl. Math. 32, 121-251 (1979).

${ }^{62}$ F. Delyon, "Apparition of purely singular continuous spectrum in a class of random Schrödinger operators," J. Stat. Phys. 40, 621-630 (1985).

${ }^{63}$ F. Delyon, Y. Levy, and B. Souillard, "Anderson localization for multidimensional systems at large disorder or large energy," Commun. Math. Phys. 100, 463-470 (1985).

${ }^{64}$ F. Delyon, B. Simon, and B. Souillard, "From power pure point to continuous spectrum in disordered systems," Ann. Inst. Henri Poincaré Phys. Theor. 42, 283-309 (1985).

${ }^{65}$ J. Derezinski, “Asymptotic completeness of long-range $N$-body quantum systems," Ann. Math. 138, 427-476 (1993).

${ }^{66}$ J. Derezinski and C. Gérard, Scattering Theory of Classical and Quantum N-Particle Systems (Springer, Berlin, 1997).

${ }^{67}$ E. Dinaburg and Ya. Sinai, “The one-dimensional Schrödinger equation with a quasiperiodic potential," Funct. Anal. Appl. 9, 279-289 (1975).

${ }^{68}$ R. K. Dodd, J. C. Eilbeck, J. D. Gibbon, and H. C. Morris, Solitons and Nonlinear Wave Equations (Academic, London, 1982).

${ }^{69}$ J. Dollard, “Asymptotic convergence and the Coulomb interaction," J. Math. Phys. 5, 729-738 (1964).

${ }^{70}$ B. A. Dubrovin, V. B. Matveev, and S. P. Novikov, "Nonlinear equations of Korteweg-de Vries type, finite-zone linear operators, and Abelian varieties," Russ. Math. Surveys 31:1, 59-146 (1976).

${ }^{71}$ F. Dyson, "Ground-state energy of a finite system of charged particles," J. Math. Phys. 8, 1538-1545 (1967).

${ }^{72}$ F. Dyson and A. Lenard, "Stability of matter, I," J. Math. Phys. 8, 423-434 (1967).

${ }^{73}$ F. Dyson and A. Lenard, "Stability of matter, II," J. Math. Phys. 9, 698-711 (1968).

${ }^{74}$ V. N. Efimov, "Energy levels arising from resonant two-body forces in a three-body system," Phys. Lett. B33, 563-564 (1970). 
${ }^{75}$ V. N. Efimov, "Weakly-bound states of three resonantly-interacting particles," Sov. J. Nucl. Phys. 12, 589-595 (1971).

${ }^{76}$ V. Enss, “A note on Hunziker's theorem," Commun. Math. Phys. 52, 233-238 (1977).

${ }^{77}$ V. Enss, “Asymptotic completeness for quantum-mechanical potential scattering, I. Short-range potentials," Commun. Math. Phys. 61, 285-291 (1978).

${ }^{78}$ V. Enss, "Completeness of three-body quantum scattering," in Dynamics and Processes, edited by P. Blanchard and L. Streit, Lecture Notes in Math, Vol. 1031 (Springer, Berlin, 1983), pp. 62-88.

${ }^{79}$ L. Faddeev, Mathematical Aspects of the Three Body Problem in Quantum Scattering Theory (Steklov Institute, 1963).

${ }^{80} \mathrm{~W}$. Faris and R. Lavine, "Commutators and self-adjointness of Hamiltonian operators," Commun. Math. Phys. 35, 39-48 (1974).

${ }^{81}$ C. Fefferman, J. Fröhlich, and G. M. Graf, "Stability of ultraviolet-cutoff quantum electrodynamics with non-relativistic matter,' Commun. Math. Phys. 190, 309-330 (1997).

${ }^{82}$ C. Fefferman and L. Seco, "On the energy of a large atom," Bull. Am. Math. Soc. 23, 525-530 (1990).

${ }^{83}$ R. Froese, "Asymptotic distribution of resonances in one dimension," J. Diff. Eqns. 137, 251-272 (1997).

${ }^{84}$ R. Froese, "Upper bounds for the resonance counting function in odd dimension," Can. J. Math. 50, 538-546 (1998).

${ }^{85}$ R. Froese and I. Herbst, “'A new proof of the Mourre estimate,' Duke Math. J. 49, 1075-1085 (1982).

${ }^{86}$ R. Froese, I. Herbst, M. Hoffmann-Ostenhof, and T. Hoffmann-Ostenhof, " $L^{2}$-exponential lower bounds to solutions of the Schrödinger equation," Commun. Math. Phys. 87, 265-286 (1982/83).

${ }^{87}$ J. Fröhlich and T. Spencer, "Absence of diffusion in the Anderson tight binding model for large disorder or low energy," Commun. Math. Phys. 88, 151-184 (1983).

${ }^{88}$ L. Garding, "Eigenfunction expansions connected with elliptic differential operators," Tolfte Skend. Mat. Lund , 44-45 (1953).

${ }^{89}$ I. Gel'fand, 'Expansion in characteristic functions of an equation with periodic coefficients," Dokl. Akad. Nauk SSSR 73, 1117-1120 (1950).

${ }^{90}$ I. M. Gel'fand and B. M. Levitan, "On the determination of a differential equation from its special function," Izv. Akad. Nauk SSSR Ser. Mat. 15, 309-360 (1951) (Russian); [Amer. Math. Soc. Transl. Ser. 2 1, 253-304 (1955)].

${ }^{91}$ F. Gesztesy and B. Simon, “A short proof of Zheludev's theorem,'” Trans. Am. Math. Soc. 335, 329-340 (1993).

${ }^{92}$ F. Gesztesy and B. Simon, "The xi function,” Acta Math. 176, 49-71 (1996).

${ }^{93}$ F. Gesztesy and B. Simon, "A new approach to inverse spectral theory, II. General real potentials and the connection to the spectral measure," Ann. Math. (to be published).

${ }^{94}$ F. Gesztesy and B. Simon, "On local Borg-Marchenko uniqueness results," Commun. Math. Phys. (to be published).

${ }^{95}$ D. J. Gilbert, "On subordinacy and analysis of the spectrum of Schrödinger operators with two singular endpoints," Proc. R. Soc. Edinburgh, Sect. A: Math. 112, 213-229 (1989).

${ }^{96}$ D. J. Gilbert, "On subordinacy and spectral multiplicity for a class of singular differential operators," Proc. R. Soc. Edinburgh, Sect. A: Math. 128, 549-584 (1998).

${ }^{97}$ D. J. Gilbert and D. B. Pearson, "On subordinacy and analysis of the spectrum of one-dimensional Schrödinger operators,"' J. Math. Anal. Appl. 128, 30-56 (1987).

${ }^{98}$ I. Goldsheid, S. Molchanov, and L. Pastur, "A pure point spectrum of the stochastic one-dimensional Schrödinger equation," Funct. Anal. Appl. 11, 1-10 (1977).

${ }^{99}$ A. Gordon, “'On the point spectrum of one-dimensional Schrödinger operators," Usp. Mat. Nauk 31, 257-258 (1976).

${ }^{100}$ A. Gordon, S. Jitomirskaya, Y. Last, and B. Simon, "Duality and singular continuous spectrum in the almost Mathieu equation," Acta Math. 178, 169-183 (1997).

${ }^{101}$ G. M. Graf, "Asymptotic completeness for N-body short-range quantum systems: A new proof," Commun. Math. Phys. 132, 73-101 (1990).

${ }^{102}$ S. Graffi, V. Grecchi, and B. Simon, “Borel summability: Application to the anharmonic oscillator,' Phys. Lett. 32B, 631-634 (1970).

${ }^{103}$ M. N. Hack, "Wave operators in multichannel scattering," Nuovo Cimento 13, 231-236 (1959).

${ }^{104}$ G. Hagedorn, "Semiclassical quantum mechanics, I. The $\hbar \rightarrow 0$ limit for coherent states," Commun. Math. Phys. 71, 77-93 (1980).

${ }^{105}$ G. Hagedorn, Semiclassical Quantum Mechanics, II. The Large Mass Asymptotics for Coherent States (Pitman, Boston, 1981).

${ }^{106}$ G. Hagedorn, "Semiclassical quantum mechanics, III. The large order asymptotics and more general states," Ann. Phys. (N.Y.) 135, 58-70 (1981).

${ }^{107}$ E. Harrell and B. Simon, "The mathematical theory of resonances which have exponentially small widths," Duke Math. J. 47, 845-902 (1980).

${ }^{108}$ B. Helffer, M. Hoffmann-Ostenhof, T. Hoffmann-Ostenhof, and M. Owen, "Nodal sets for groundstates of Schrödinger operators with zero magnetic field in non-simply connected domains," Commun. Math. Phys. 202, 629-649 (1999).

${ }^{109}$ B. Helffer and D. Robert, "Riesz means of bounded states and semi-classical limit connected with a Lieb-Thirring conjecture," Asymp. Anal. 3, 91-103 (1990).

${ }^{110}$ B. Helffer and D. Robert, "Riesz means of bounded states and semi-classical limit connected with a Lieb-Thirring conjecture, II,” Ann. Inst. Henri Poincaré Phys. Theor. 53, 139-147 (1990).

${ }^{111}$ B. Helffer and J. Sjöstrand, "Multiple wells in the semiclassical limit, I," Commun. Partial Diff. Eqns. 9, 337-408 (1984).

${ }^{112}$ B. Helffer and J. Sjöstrand, "Résonances en limite semi-classique," Mém. Soc. Math. France (N.S.) No. 24, 24-25 (1986).

${ }^{113}$ R. Hempel and J. Voight, "The spectrum of a Schrödinger operator in $L^{p}\left(\mathbb{R}^{\nu}\right)$ is $p$-independent," Commun. Math. Phys. 104, 243-250 (1986). 
${ }^{114}$ I. Herbst, “Unitary equivalences of Stark-Hamiltonians,” Math. Z. 155, 55-70 (1977).

${ }^{115}$ I. Herbst, "Dilation analyticity in constant electric field, I. The two-body problem," Commun. Math. Phys. 64, 279298 (1979).

${ }^{116}$ I. Herbst, J. Møller, and E. Skibsted, “Asymptotic completeness for $N$-body Stark Hamiltonians,” Commun. Math. Phys. 174, 509-535 (1996).

${ }^{117}$ I. Herbst and B. Simon, "Some remarkable examples in eigenvalue perturbation theory," Phys. Lett. 78B, 304-306 (1978).

${ }^{118}$ I. Herbst and S. Sloan, "Perturbations of translation invariant positivity preserving semigroups in $L^{2}(R)$, , Trans. Am. Math. Soc. 236, 325-360 (1978).

${ }^{119}$ H. Hess, R. Schrader, and D. Uhlenbrock, “Domination of semigroups and generalization of Kato's inequality," Duke Math. J. 44, 893-904 (1977).

${ }^{120}$ M. Hoffmann-Ostenhof, T. Hoffmann-Ostenhof, and N. Nadirashvili, “'Interior Hölder estimates for solutions of Schrödinger equations and the regularity of nodal sets," Commun. Partial Diff. Eqns. 20, 1241-1273 (1995).

${ }^{121}$ M. Hoffmann-Ostenhof, T. Hoffmann-Ostenhof, and H. Stremmnitzer, "Local properties of Coulombic wave functions," Commun. Math. Phys. 163, 185-215 (1994).

${ }^{122}$ L. Hörmander, The Analysis of Linear Partial Differential Operators, IV. Fourier Integral Operators (Springer, Berlin, 1994).

${ }^{123}$ W. Hughes, "An atomic energy lower bound that agrees with Scott's correction," Adv. Math. 79, 213-270 (1990).

${ }^{124}$ D. Hundertmark, A. Laptev, and T. Weidl, "New bounds on the Lieb-Thirring constants," Invent. Math. (to be published).

${ }^{125}$ D. Hundertmark, E. Lieb, and L. Thomas, "A sharp bound for an eigenvalue moment of the one-dimensional Schrödinger operator," Adv. Theor. Math. Phys. 2, 719-731 (1998).

${ }^{126}$ W. Hunziker, "On the spectra of Schrödinger multiparticle Hamiltonians," Helv. Phys. Acta 39, 451-462 (1966).

${ }^{127}$ W. Hunziker, "Mathematical theory of $N$-body quantum systems," Helv. Phys. Acta 71, 26-43 (1998).

${ }^{128}$ W. Hunziker and I. M. Sigal, "The quantum $N$-body problem," preprint.

${ }^{129}$ T. Ikebe, "Eigenfunction expansions associated with the Schrödinger operators and their application to scattering theory," Arch. Ration. Mech. Anal. 5, 1-34 (1960).

${ }^{130}$ T. Ikebe and T. Kato, "Uniqueness of the self-adjoint extension of singular elliptic differential operators," Arch. Ration. Mech. Anal. 9, 77-92 (1962).

${ }^{131}$ R. Iorio and M. O'Carroll, "Asymptotic completeness for the multi-particle Schrödinger Hamiltonians with weak potentials," Commun. Math. Phys. 27, 137-145 (1972).

${ }^{132}$ A. R. Its and V. B. Matveev, "Schrödinger operators with finite-gap spectrum and $N$-soliton solutions of the Korteweg-de Vries equation," Theor. Math. Phys. 23, 343-355 (1975).

${ }^{133}$ J. M. Jauch, “Theory of the scattering operator," Helv. Phys. Acta 31, 127-158 (1958).

${ }^{134}$ S. Jitomirskaya, "Metal-insulator transition for the almost Mathieu operator," Ann. Math. (to be published).

${ }^{135}$ S. Jitomirskaya and Y. Last, "Dimensional Hausdorff properties of singular continuous spectra," Phys. Rev. Lett. 76, 1765-1769 (1996).

${ }^{136}$ S. Jitomirskaya and B. Simon, “'Operators with singular continuous spectrum, III. Almost periodic Schrödinger operators," Commun. Math. Phys. 165, 201-205 (1994).

${ }^{137}$ G. Kac, 'Expansion in characteristic functions of self-adjoint operators,' Dokl. Akad. Nauk SSSR 119, 19-22 (1958).

${ }^{138}$ I. S. Kac, "On the multiplicity of the spectrum of a second-order differential operator," Sov. Math. Dokl. 3, 1035-1039 (1962).

${ }^{139}$ I. S. Kac, "Spectral multiplicity of a second-order differential operator and expansion in eigenfunction," Izv. Akad. Nauk SSSR, Ser. Mat. 27, 1081-1112 (1963).

${ }^{140}$ M. Kac, "On some connections between probability theory and differential equations," Proceedings 2nd Berkeley Symposium on Mathematical Statistics and Probability, University of California Press, Berkeley and Los Angeles, 1951, pp. $189-215$.

${ }^{141}$ M. Kac, "On the asymptotic number of bound states for certain attractive potentials" in Topics in Functional Analysis, edited by I. Gohberg and M. Kac (Academic, New York, 1978), pp. 159-167.

${ }^{142}$ T. Kato, "On the convergence of the perturbation method, I," Prog. Theor. Phys. 4, 514-523 (1949).

${ }^{143}$ T. Kato, "On the convergence of the perturbation method, II," Prog. Theor. Phys. 5, 95-101 (1950); 5, 207-212 (1950).

${ }^{144}$ T. Kato, "Fundamental properties of Hamiltonian operators of Schrödinger type,' Trans. Am. Math. Soc. 70, 195-211 (1951).

${ }^{145}$ T. Kato, "On the existence of solutions of the helium wave equation," Trans. Am. Math. Soc. 70, 212-218 (1951).

${ }^{146}$ T. Kato, "On the convergence of the perturbation method,' J. Fac. Sci., Univ. Tokyo, Sect. I 6, 145-226 (1951).

${ }^{147}$ T. Kato, "Perturbation theory of semi-bounded operators," Math. Ann. 125, 435-447 (1953).

${ }^{148}$ T. Kato, "On the eigenfunctions of many particle systems in quantum mechanics," Commun. Pure Appl. Math. 10, $151-171$ (1957).

${ }^{149}$ T. Kato, "Perturbation of continuous spectra by trace class operators," Proc. Jpn. Acad. 33, 260-264 (1957).

${ }^{150}$ T. Kato, "Growth properties of solutions of the reduced wave equation with variable coefficients," Commun. Pure Appl. Math. 12, 403-425 (1959).

${ }^{151}$ T. Kato, "Schrödinger operators with singular potentials," Isr. J. Math. 13, 135-148 (1972).

${ }^{152}$ T. Kato, "Remarks on Schrödinger operators with vector potentials," Int. Equ. Operator Theory 1, 103-113 (1978).

${ }^{153}$ T. Kato, Perturbation Theory for Linear Operators, 2nd ed. (Springer, Berlin, 1980).

${ }^{154}$ C. E. Kenig, Harmonic Analysis Techniques for Second Order Elliptic Boundary Value Problems (American Mathematical Society, Providence, RI, 1994). 
${ }^{155}$ R. Killip, "Perturbations of one-dimensional Schrödinger operators preserving the a.c. spectrum," preprint.

${ }^{156}$ A. Kiselev, "Absolutely continuous spectrum of one-dimensional Schrödinger operators and Jacobi matrices with slowly decreasing potentials,' Commun. Math. Phys. 179, 377-400 (1996).

${ }^{157}$ A. Kiselev, "Stability of the absolutely continuous spectrum of the Schrödinger equation under slowly decaying perturbations and a.e. convergence of integral operators," Duke Math. J. 94, 619-646 (1998).

${ }^{158}$ A. Kiselev, Y. Last, and B. Simon, "Modified Prüfer and EFGP transforms and the spectral analysis of one-dimensional Schrödinger operators," Commun. Math. Phys. 194, 1-45 (1998).

${ }^{159}$ S. Kotani, "Lyapunov exponents and spectra for one-dimensional random Schrödinger operators," Contemporary Mathematics (American Mathematical Society, Providence, RI, 1986), Vol. 50.

${ }^{160}$ S. Kotani and N. Ushiroya, "One-dimensional Schrödinger operators with random decaying potentials," Commun. Math. Phys. 115, 247-266 (1988).

${ }^{161} \mathrm{~V}$. Kovalenko and Yu. Semenov, "Some problems on expansions in generalized eigenfunctions of the Schrödinger operator with strongly unique potentials," Rus. Math. Surveys 33, 119-157 (1978).

${ }^{162}$ M. G. Krein, "'Solution of the inverse Sturm-Liouville problem," Dokl. Akad. Nauk SSSR 76, 21-24 (1951).

${ }^{163}$ M. G. Krein, "Determination of the density of a nonhomogeneous symmetric cord by its frequency spectrum," Dokl. Akad. Nauk SSSR 76, 345-348 (1951).

${ }^{164}$ M. G. Krein, "On determination of the potential of a particle from its $S$-function," Dokl. Akad. Nauk SSSR 105, 433-436 (1955).

${ }^{165}$ H. Kunz and B. Souillard, “Sur le spectre des operateurs aux differences finies aleatoires," Commun. Math. Phys. 78, 201-246 (1980).

${ }^{166}$ S. Kuroda, "Perturbations of continuous spectra by unbounded operators, I," J. Math. Soc. Jpn. 11, 247-262 (1959).

${ }^{167}$ S. Kuroda, "Perturbations of continuous spectra by unbounded operators, II," J. Math. Soc. Jpn. 12, $243-257$ (1960).

${ }^{168}$ A. Laptev and T. Weidl, "Sharp Lieb-Thirring inequalities in high dimensions," Acta Math 184, 87-111 (2000).

${ }^{169}$ Y. Last, "A relation between a.c. spectrum of ergodic Jacobi matrices and the spectra of periodic approximants," Commun. Math. Phys. 151, 183-192 (1993).

${ }^{170}$ Y. Last and B. Simon, "Eigenfunctions, transfer matrices, and absolutely continuous spectrum of one-dimensional Schrödinger operators," Invent. Math. 135, 329-367 (1999).

${ }^{171}$ R. Lavine, "Absolute continuity of Hamiltonian operators with repulsive potentials," Proc. Am. Math. Soc. 22, 55-60 (1969).

${ }^{172}$ R. Lavine, “Absolute continuity of positive spectrum for Schrödinger operators with long range potentials," J. Funct. Anal. 12, 30-54 (1973).

${ }^{173}$ H. Leinfelder and C. Simader, "Schrödinger operators with singular magnetic vector potentials," Math. Z. 176, 1-19 (1981).

${ }^{174}$ B. Ya. Levin, "Fourier- and Laplace-type transformations by means of solutions of a second-order differential equation," Dokl. Akad. Nauk SSSR 106, 187-190 (1956).

${ }^{175}$ B. M. Levitan, Theory of Generalized Translation Operators, 2nd ed. (Nauka, Moscow, 1973).

${ }^{176}$ B. M. Levitan, Inverse Sturm-Liouville Problems (VNU Science, Utrecht, 1987).

${ }^{177}$ E. Lieb, "Bounds on the eigenvalues of the Laplace and Schrödinger operators," Bull. Am. Math. Soc. 82, 751-753 (1976).

${ }^{178}$ E. Lieb, "Bound on the maximum negative ionization of atoms and molecules," Phys. Rev. A 29, 3018-3028 (1984).

${ }^{179}$ E. Lieb, "The stability of matter: From atoms to stars," Bull. Am. Math. Soc. 22, 1-49 (1990).

${ }^{180}$ E. Lieb, M. Loss, and J. P. Solovej, "Stability of matter in magnetic fields," Phys. Rev. Lett. 75, 985-989 (1995).

${ }^{181}$ E. Lieb, I. M. Sigal, B. Simon, and W. Thirring, “'Asymptotic neutrality of large Z ions," Phys. Rev. Lett. 52, 994-996 (1984).

${ }^{182}$ E. Lieb and B. Simon, "The Thomas-Fermi theory of atoms, molecules, and solids," Adv. Math. 23, 22-116 (1977).

${ }^{183}$ E. Lieb, J. P. Solovej, and J. Yngvason, “Asymptotics of heavy atoms in high magnetic fields, I. Lowest Landau band regions," Commun. Pure Appl. Math. 47, 513-591 (1994).

${ }^{184}$ E. Lieb, J. P. Solovej, and J. Yngvason, "Asymptotics of heavy atoms in high magnetic fields, II. Semiclassical regions," Commun. Math. Phys. 161, 77-124 (1994).

${ }^{185}$ E. Lieb and W. Thirring, "Bound for the kinetic energy of fermions which proves the stability of matter," Phys. Rev. Lett. 35, 687-689 (1975).

${ }^{186} \mathrm{E}$. Lieb and W. Thirring, "Inequalities for the moments of the eigenvalues of the Schrödinger Hamiltonian and their relation to Sobolev inequalities," in Studies in Mathematical Physics: Essays in Honor of Valentine Bargmann, edited by E. Lieb, B. Simon, and A. Wightman (Princeton University Press, Princeton, 1976), pp. 269-303.

${ }^{187}$ J. Loeffel, A. Martin, B. Simon, and A. Wightman, "Padé approximants and the anharmonic oscillator," Phys. Lett. 30B, 656-658 (1969).

${ }^{188}$ V. A. Marchenko, "Certain problems in the theory of second-order differential operators," Dokl. Akad. Nauk SSSR 72, 457-460 (1950).

${ }^{189} \mathrm{~V}$. A. Marchenko, "Some questions in the theory of one-dimensional linear differential operators of the second order, I,’ Am. Math. Soc. Transl. 101, 1-104 (1973).

${ }^{190}$ V. A. Marchenkov, Sturm-Liouville Operators and Applications (Birkhäuser, Basel, 1986).

${ }^{191}$ A. Martin, “'Bound states in the strong coupling limit," Helv. Phys. Acta 45, 140-148 (1972).

${ }^{192}$ H. McKean and E. Trubowitz, "Hill's operator and hypoelliptic function theory in the presence of infinitely many branch points," Commun. Pure Appl. Math. 29, 143-226 (1976).

${ }^{193}$ H. McKean and P. van Moerbeke, “The spectrum of Hill's equation,”' Invent. Math. 30, 217-274 (1975).

${ }^{194}$ J. B. McLeod, "The distribution of eigenvalues for the hydrogen atom and similar cases," Proc. London Math. Soc. 11, $139-158(1961)$. 
${ }^{195}$ A. Melin, “'Operator methods for inverse scattering on the real line," Commun. Partial Diff. Eqns. 10, 677-766 (1985).

${ }^{196}$ E. Mourre, "Absence of singular continuous spectrum for certain selfadjoint operators," Commun. Math. Phys. 78, 391-408 (1981).

${ }^{197}$ S. N. Naboko, "On the dense point spectra of Schrödinger and Dirac operators," Theor. Math. Phys. 68, 18-28 (1986).

${ }^{198}$ E. Nelson (unpublished).

${ }^{199}$ S. Novikov, S. V. Manakov, L. P. Pitaevskii, and V. E. Zakharov, Theory of Solitons (Consultants Bureau, New York, 1984).

${ }^{200}$ A. O'Connor, "Exponential decay of bound state wave functions," Commun. Math. Phys. 32, 319-340 (1973).

${ }^{201}$ Yu. Ovchinnikov and I. M. Sigal, "Number of bound states of three-body systems and Efimov's effect," Ann. Phys. (N.Y.) 123, 274-295 (1979).

${ }^{202}$ L. Pastur, "Spectral properties of disordered systems in the one-body approximation," Commun. Math. Phys. 75, 179-196 (1980).

${ }^{203}$ L. Pastur and A. Figotin, Spectra of Random and Almost-Periodic Schrödinger Operators (Springer, Berlin, 1992).

${ }^{204}$ D. B. Pearson, "Singular continuous measures in scattering theory," Commun. Math. Phys. 60, 13-36 (1978).

${ }^{205}$ P. Perry, I. M. Sigal, and B. Simon, "Spectral analysis of multiparticle Schrödinger operators," Ann. Math. 114, 519-567 (1981).

${ }^{206}$ J. Piepenbrink, “Nonoscillatory elliptic equations," J. Diff. Eqns. 15, 541-550 (1974).

${ }^{207}$ Y. Pinchover, “'On positive solutions of second-order elliptic equations, stability results and classification,' Duke Math. J. 57, 955-980 (1988).

${ }^{208}$ A. Ya. Povzner, "On differential equations of Sturm-Liouville type on a half-axis," Mat. Sb. 23, 3-52 (1948) (Russian); [Am. Math. Soc. Transl. 4, 24-101 (1962)].

${ }^{209}$ A. Ya. Povzner, "On the expansion of arbitrary functions in terms of the eigenfunctions of the operator $-\Delta u+c u$," Mat. Sb. 43, 109-156 (1953), (Russian); [Am. Math. Soc. Transl. 60, 268-302 (1967)].

${ }^{210}$ J. Ralston, "On the construction of quasimodes associated with stable periodic orbits," Commun. Math. Phys. 51 219-242 (1976), 67, 91 (1979) (Erratum).

${ }^{211}$ M. Reed and B. Simon, Methods of Modern Mathematical Physics, II. Fourier Analysis, Self-Adjointness (Academic, New York, 1975).

${ }^{212}$ M. Reed and B. Simon, Methods of Modern Mathematical Physics, IV. Analysis of Operators (Academic, New York, 1978).

${ }^{213}$ M. Reed and B. Simon, Methods of Modern Mathematical Physics, III. Scattering Theory (Academic, New York, 1979).

${ }^{214}$ M. Reed and B. Simon, Methods of Modern Mathematical Physics, I. Functional Analysis, rev. ed. (Academic, New York, 1980).

${ }^{215}$ F. Rellich, 'Störungstheorie der Spektralzerlegung I-V,’’ Math. Ann. 113, 600-619 (1937); 113, 677-685 (1937); 116, 555-570 (1939); 117, 356-382 (1940); 118, 462-484 (1942).

${ }^{216}$ F. Rellich, "Über das asymptotische Verhalten der Lösungen von $\Delta u+\lambda u=0$ in unendlichen Gebieten," Jber. Deutsch. Math. Verein 53, 57-65 (1943).

${ }^{217}$ C. Remling, "A probabilistic approach to one-dimensional Schrödinger operators with sparse potentials," Commun. Math. Phys. 185, 313-323 (1997).

${ }^{218}$ C. Remling, "The absolutely continuous spectrum of one-dimensional Schrödinger operators with decaying potentials," Commun. Math. Phys. 193, 151-170 (1998).

${ }^{219}$ D. Robert, "Semi-classical approximation in quantum mechanics. A survey of old and recent mathematical results," Helv. Phys. Acta 71, 44-116 (1998).

${ }^{220}$ G. V. Rosenbljum, "The distribution of the discrete spectrum for singular differential operators," Dokl. Akad. Nauk SSSR 202, 1012-1015 (1972) (Russian); [Sov. Math. Dokl. 13, 245-249 (1972)].

${ }^{221}$ M. Rosenblum, "Perturbations of continuous spectrum and unitary equivalence," Pac. J. Math. 7, 997-1010 (1957).

${ }^{222}$ D. Ruelle, "A remark on bound states in potential scattering theory," Nuovo Cimento A 61A, 655-662 (1969).

${ }^{223}$ M. B. Ruskai, "Absence of discrete spectrum in highly negative ions," Commun. Math. Phys. 82, 457-469 (1982).

${ }^{224}$ M. B. Ruskai, “Absence of discrete spectrum in highly negative ions, II," Commun. Math. Phys. 85, 325-327 (1982).

${ }^{225}$ A. Sá Barreto and M. Zworski, "Existence of resonances in potential scattering," Commun. Pure Appl. Math. 49, 1271-1280 (1996).

${ }^{226}$ M. Schechter, Spectra of Partial Differential Operators (North Holland, Amsterdam, 1971).

${ }^{227}$ J. Schwinger, "On the bound states of a given potential," Proc. Natl. Acad. Sci. USA 47, 122-129 (1961).

${ }^{228}$ C. Shubin, R. Vakilian, and T. Wolff, "Some harmonic analysis questions suggested by Anderson-Bernoulli models," Geom. Funct. Anal. 8, 932-964 (1998).

${ }^{229} \mathrm{H}$. Siedentop and R. Weikard, "On the leading energy correction for the statistical model of the atom: Interacting case," Commun. Math. Phys. 112, 471-490 (1987).

${ }^{230} \mathrm{H}$. Siedentop and R. Weikard, "On the leading correction of the Thomas-Fermi model: Lower bound," Invent. Math. 97, 159-193 (1989).

${ }^{231}$ I. M. Sigal, "Geometric methods in the quantum many-body problem. Nonexistence of very negative ions," Commun. Math. Phys. 85, 309-324 (1982).

${ }^{232}$ I. M. Sigal, "How many electrons can a nucleus bind?," Ann. Phys. (N.Y.) 157, 307-320 (1984).

${ }^{233}$ I. M. Sigal and A. Soffer, "The $N$-particle scattering problem: Asymptotic completeness for short-range systems," Ann. Math. 126, 35-108 (1987).

${ }^{234}$ I. M. Sigal and A. Soffer, "Asymptotic completeness of $N$-particle long-range scattering," J. Am. Math. Soc. 7, 307-334 (1994) 
${ }^{235}$ B. Simon, "On positive eigenvalues of one-body Schrödinger operators," Commun. Pure Appl. Math. 22, 531-538 (1969).

${ }^{236} \mathrm{~B}$. Simon, "On the infinitude or finiteness of the number of bound states of an $N$-body quantum system, I,' Helv. Phys. Acta 43, 607-630 (1970).

${ }^{237}$ B. Simon, "Quadratic form techniques and the Balslev-Combes theorem," Commun. Math. Phys. 27, 1-9 (1972).

${ }^{238} \mathrm{~B}$. Simon, "Resonances in $N$-body quantum systems with dilation analytic potentials and the foundations of timedependent perturbation theory," Ann. Math. 97, 247-274 (1973).

${ }^{239}$ B. Simon, "Essential self-adjointness of Schrödinger operators with positive potentials," Math. Ann. 201, 211-220 (1973).

${ }^{240}$ B. Simon, "On the number of bound states of two-body Schrödinger operators-A review," in Studies in Mathematical Physics: Essays in Honor of Valentine Bargmann, edited by E. Lieb, B. Simon, and A. Wightman (Princeton University Press, Princeton, 1976), pp. 305-326.

${ }^{241}$ B. Simon, "Universal diamagnetism of spinless Bose systems," Phys. Rev. Lett. 36, 1083-1084 (1976).

${ }^{242}$ B. Simon, "The bound state of weakly coupled Schrödinger operators in one and two dimensions," Ann. Phys. (N.Y.) 97, 279-288 (1976).

${ }^{243}$ B. Simon, "Analysis with weak trace ideals and the number of bound states of Schrödinger operators," Trans. Am. Math. Soc. 224, 367-380 (1976).

${ }^{244}$ B. Simon, "Geometric methods in multiparticle quantum systems," Commun. Math. Phys. 55, 259-274 (1977).

${ }^{245}$ B. Simon, “Complex scaling: A rigorous overview," Int. J. Quantum Chem. 14, 529-542 (1978).

${ }^{246}$ B. Simon, Functional Integration and Quantum Physics (Academic, New York, 1979).

${ }^{247}$ B. Simon, "Kato's inequality and the comparison of semigroups," J. Funct. Anal. 32, 97-101 (1979).

${ }^{248}$ B. Simon, "Maximal and minimal Schrödinger forms," J. Oper. Th. 1, 37-47 (1979).

${ }^{249}$ B. Simon, "Phase space analysis of simple scattering systems. Extensions of some work of Enss," Duke Math. J. 46, 119-168 (1979).

${ }^{250}$ B. Simon, “Schrödinger semigroups," Bull. Am. Math. Soc. 7, 447-526 (1982).

${ }^{251}$ B. Simon, "Some Jacobi matrices with decaying potential and dense point spectrum," Commun. Math. Phys. 87, 253-258 (1982).

${ }^{252}$ B. Simon, "Semiclassical analysis of low lying eigenvalues, I. Non-degenerate minima: Asymptotic expansions," Ann. Inst. Henri Poincaré Phys. Theor. 38, 295-307 (1983).

${ }^{253}$ B. Simon, "Semiclassical analysis of low lying eigenvalues, II. Tunneling," Ann. Math. 120, 89-118 (1984).

${ }^{254}$ B. Simon, "Fifty years of eigenvalue perturbation theory," Bull. Am. Math. Soc. 24, 303-319 (1991).

${ }^{255}$ B. Simon, "Operators with singular continuous spectrum, I. General operators," Ann. Math. 141, 131-145 (1995).

${ }^{256}$ B. Simon, "Bounded eigenfunctions and absolutely continuous spectra for one-dimensional Schrödinger operators," Proc. Am. Math. Soc. 124, 3361-3369 (1996).

${ }^{257}$ B. Simon, "Some Schrödinger operators with dense point spectrum," Proc. Am. Math. Soc. 125, 203-208 (1997).

${ }^{258}$ B. Simon, "A new approach to inverse spectral theory, I. Fundamental formalism," Ann. Math. 150, 1-29 (1999).

${ }^{259}$ B. Simon, “A Feynman-Kac formula for unbounded semigroups," Proceedings International Conference on Infinite Dimensional (Stochastic) Analysis and Quantum Physics, Leipzig, 1999 (to be published).

${ }^{260}$ B. Simon, "Schrödinger Operators in the Twenty-First Century,"' Mathematical Physics 2000 (Imperial College Press, London, to be published).

${ }^{261}$ B. Simon and T. Wolff, "Singular continuous spectrum under rank one perturbations and localization for random Hamiltonians," Commun. Pure Appl. Math. 39, 75-90 (1986).

${ }^{262}$ F. Stummel, "Singuläre elliptische Differentialoperatoren in Hilbertschen Räumen," Math. Ann. 132, 150-176 (1956).

${ }^{263}$ H. Tamura, "The asymptotic eigenvalue distribution for nonsmooth elliptic operators," Proc. Jpn. Acad. 50, 19-22 (1974).

${ }^{264}$ L. Thomas, "Time dependent approach to scattering from impurities in a crystal," Commun. Math. Phys. 33, 335-343 (1973).

${ }^{265}$ E. C. Titchmarsh, "Some theorems on perturbation theory, I," Proc. R. Soc. London, Ser. A 200, 34-46 (1949).

${ }^{266}$ E. C. Titchmarsh, "Some theorems on perturbation theory, II," Proc. R. Soc. London, Ser. A 201, $473-479$ (1950).

${ }^{267}$ E. C. Titchmarsh, Eigenfunction Expansions, Part I, II, (Oxford University Press, London), Part I, 2nd ed. (1962); Part II (1958).

${ }^{268}$ E. Trubowitz, “The inverse problem for periodic potentials," Commu. Pure Appl. Math 30, 321-337 (1977).

${ }^{269}$ C. van Winter, “Theory of finite systems of particles I,” Mat. Fys. Skr. Danske Vid. Selsk. 1, 1-60 (1964).

${ }^{270} \mathrm{H}$. von Dreifus and A. Klein, "A new proof of localization in the Anderson tight binding model," Commun. Math. Phys. 124, 285-299 (1989).

${ }^{271}$ J. von Neumann, Mathematical Foundations of Quantum Mechanics (Springer, Berlin, 1932).

${ }^{272}$ J. von Neumann and E. Wigner, “Über merkwürdige diskrete Eigenwerte," Z. Phys. 30, 465-467 (1929).

${ }^{273}$ J. Weidmann, “'Zur Spektraltheorie von Sturm-Liouville-Operatoren," Math. Z. 98, 268-302 (1967).

${ }^{274} \mathrm{H}$. Weyl, "Das asymptotische Verteilungsgesetz der Eigenwerte linearer partieller Differentialgleichungen," Math. Ann. 71, 441-469 (1911)

${ }^{275} \mathrm{H}$. Weyl, The Theory of Groups and Quantum Mechanics (Dover, New York, 1931).

${ }^{276}$ D. R. Yafaev, "'On the theory of the discrete spectrum of the three-particle Schrödinger operator," Math. USSR Sb. 23, 535-559 (1974).

${ }^{277}$ D. R. Yafaev, Mathematical Scattering Theory (American Mathematical Society, Providence, RI, 1992).

${ }^{278}$ D. R. Yafaev, "Radiation conditions and scattering theory for $N$-particle Hamiltonians," Commun. Math. Phys. 154 523-554 (1993) 
${ }^{279}$ K. Yajima, "Spectral and scattering theory for Schrödinger operators with Stark-effect," J. Fac. Sci., Univ. Tokyo, Sect. 1 26, 377-390 (1979).

${ }^{280}$ G. M. Zhislin, "Discussion of the spectrum of Schrödinger operator for systems of many particles," Tr. Mosk. Mat. Obs. 9, 81-128 (1960).

${ }^{281} \mathrm{G}$. M. Zhislin, "On the finiteness of the discrete spectrum of the energy operator of negative atomic and molecular ions,” Teor. Mat. Fiz. 7, 571-578 (1971).

${ }^{282}$ M. Zworski, "Distribution of poles for scattering on the real line," J. Funct. Anal. 73, 277-296 (1987).

${ }^{283}$ M. Zworski, "Sharp polynomial bounds on the number of scattering poles," Duke Math. J. 59, 311-323 (1989).

${ }^{284}$ M. Zworski, "Counting scattering poles," in Spectral and Scattering Theory, edited by M. Ikawa (Marcel Dekker, New York, 1994), pp. 301-331.

${ }^{285}$ M. Zworski, “Resonances in physics and geometry," Notices Am. Math. Soc. 46, 319-328 (1999). 